\title{
Simple Graphs in Granular Computing ${ }^{1}$
}

\author{
Giampiero Chiaselotti ${ }^{\mathrm{a}}$, Davide Ciucci ${ }^{\mathrm{b}}$, Tommaso Gentile $\mathrm{e}^{\mathrm{a}, \mathrm{c}}$ \\ ${ }^{a}$ Dipartimento di Matematica e Informatica, Università della Calabria \\ Via Pietro Bucci, Cubo 30B, 87036 Arcavacata di Rende (CS), Italy. \\ ${ }^{b}$ Dipartimento di Informatica, Sistemistica e Comunicazione, Università di Milano-Bicocca \\ Via Bicocca degli Arcimboldi 8 - 20126 Milano, Italy \\ ${ }^{c}$ Istituto Nazionale di Fisica Nucleare, Gruppo collegato di Cosenza, Università della Calabria \\ Via Pietro Bucci, Cubo 31C, 87036 Arcavacata di Rende (CS), Italy.
}

\begin{abstract}
Given a graph, we interpret its adjacency matrix as an information table. We study this correspondence in two directions. Firstly, on the side of graphs by applying to it standard techniques from granular computing. In this way, we are able to connect automorphisms on graphs to the so-called indiscernibility relation and a particular hypergraph built from the starting graph to core and reducts. On the other hand, new concepts are introduced on graphs that have an interesting corresponding on information tables. In particular, some new topological interpretations of the graph and the concept of extended core are given.
\end{abstract}

Key words: Granular Computing, Information Tables, Rough Set Theory, Reduct Hypergraph, Simple Graphs, Complete Graph, Complete Bipartite Graph, Petersen Graph.

\section{Introduction}

Granular computing (briefly $\mathrm{GrC}$ ) deals with representing and processing information in the form of some type of aggregates. These aggregates are generally called information granules or simply granules and they arise in the process of data abstraction and knowledge extraction from data. Generally speaking, information granules are collections of entities arranged together due to their similarity, functional or physical adjacency, indistinguishability, coherency, and so on. The scope of $\mathrm{GrC}$ covers various fields of study related to knowledge representation and extraction. In 1979 the concept of information granularity was introduced by Zadeh [52] and it was related to the research on fuzzy sets. Then, the term granular computing was used again by Zadeh in 1997 [53] with the following words: "a subset of computing with words is granular computing". Since 1979, granular computing has become a very developed area of research in the scope of both applied and theoretical information sciences [32,34]. Today $\mathrm{GrC}$ has emerged as

\footnotetext{
${ }^{1}$ Appeared in Information Sciences, 340-341, 2016, 279-304, DOI: 10.1016/j.ins.2015.12.042

Email addresses: giampiero.chiaselotti@unical.it (Giampiero Chiaselotti), ciucci@disco.unimb.it (Davide Ciucci), gentile@mat.unical.it (Tommaso Gentile)

Preprint submitted to Information Sciences

February 5, 2016
} 
a standalone research area that intersects and finds application in several fields related to knowledge management: interval analysis [22], machine learning [51], formal concept analysis [46, 21], data mining [20, 25, 26, 43, 47], database theory [19,35], rough set theory $[28,29,48]$, interactive computing $[37,38]$ and fuzzy set theory [33, 53].

In this paper we are mainly interested in the part of $\mathrm{GrC}$ related to information tables and their management by rough-set theory tools. An information table, from now on denoted as $\mathcal{J}$, is simply a bi-dimensional table associating to any object (rows of the table) a value (content of the table) for each attribute under investigation (columns). If all values are 0 or 1 , the information table is said Boolean. An information table is obviously a very common structure in various fields of study, both of qualitative and quantitative type. In his seminal works concerning rough set (RS) theory [27, 28], Pawlak introduced several investigation tools in order to analyze a generic information table and reduce its complexity. The fundamental concept that allows us to connect the Pawlak theory to the GrC paradigm is the indiscernibility relation $I_{A}$, where $A$ is any attribute subset. Very simply, two objects are $A$-indiscernible if they assume the same value for all attributes $a \in A$. Now, since $I_{A}$ is an equivalence relation, we can partition the object set in equivalence classes, i.e., in granules, and therefore we can interpret a great part of Pawlak's theory as a particular type of $\mathrm{GrC}$, from now on $\mathrm{GrC}-\mathrm{RS}$ (for a detailed analysis concerning the links between $\mathrm{GrC}$ and rough sets see [50]).

There are at least four concepts in GrC-RS that deserve a special consideration: the indiscernibility relation, the core, the reduct family and the discernibility matrix. The core [28] can be intuitively described as the most important part of the attribute set that characterizes an information table. A reduct [28] is a subset of attributes that provides sufficient information to fully characterize the entire table. Finally, the discernibility matrix [36] is a square matrix having in the place $(i, j)$ the set of attributes on which the objects $u_{i}$ and $u_{j}$ differ.

With the term granular computing on graphs and hypergraphs (abbreviated GrC$\mathrm{GH}$ ), we mean all the studies that link graph and hypergraph theory with GrC. We notice that GrC-GH is not a research sub-field of GrC-RS since one can study several types of granularity outside of rough set theory [32, 34], and we will see in sub-section 1.3 that this has indeed been done. So, here we are interested in the intersection of the two fields GrC-GH and GrC-RS. More in detail, we will interpret the adjacency matrix of a (finite) simple undirected graph $G$ as a Boolean information table $\mathcal{J}[G]$, where the object set and the attribute set coincide. In this way, we can efficiently use the theoretical framework developed in GrC-RS to find new properties concerning the graph $G$. From this point of view, the advantage is twofold: both in direction from GrC-RS towards discrete mathematics (briefly GrC-RS $\rightarrow \mathrm{DM}$ ) and also from discrete mathematics towards GrC-RS (briefly DM $\rightarrow$ GrC-RS). We will now illustrate this double advantage by providing more details about two results obtained in this paper.

\subsection{An Example of $G r C-R S \rightarrow D M$}

In the Boolean information table $\mathcal{J}[G]$, for a fixed vertex subset $A$, the indiscernibility relation $I_{A}$ is an equivalence relation between vertices of $G$ that can be characterized in geometric terms. Specifically, two vertices are $I_{A}$-indiscernible if and only if they are in a type of "symmetrical" position with respect to all the vertices of $A$. This means that we can interpret $A$ as if it were a "symmetry block" for the graph $G$ (see Theorem 3.3). On the basis of this result it is natural to ask what do the core, the reducts and 
the granular lattice for common families of graphs become. In this paper we treat the complete graphs, the complete bipartite graphs, the Petersen graph and the paths on a fixed number of vertices cases.

Let us consider for example the classical Petersen graph (briefly Pet):

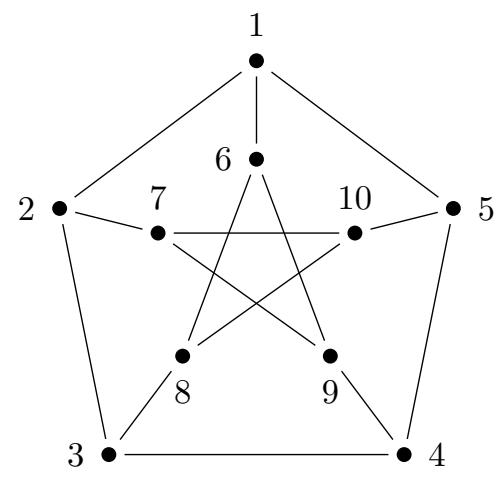

Figure 1: The Petersen graph

For its symmetry properties (the Petersen graph is both vertex-transitive and edgetransitive) the core is empty (see Corollary 6.3). However the key point, a priori not obvious, turns out to be that the reducts of the Petersen graph can be characterized by a geometric point of view. In fact, we will provide a purely combinatorial and geometric proof of the following classification result (a part of Theorem 6.6): a vertex subset $A$ of Pet is a reduct of the Boolean information table $\mathfrak{J}[$ Pet $]$ if and only if $A$ has cardinality 5 and the subgraph of Pet generated by $A$ is isomorphic to one of the following graphs:

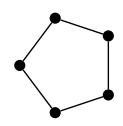

(a)

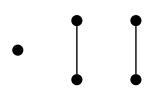

(b)

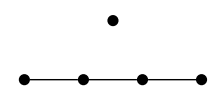

(c) (d)

Figure 2: Reducts of the Petersen Graph

This result is indeed a specific non-trivial example that shows how we can find unexpected links between the geometric structure of the graph and its interpretation as a Boolean information table subject to granular investigation.

\subsection{An Example of $D M \rightarrow G r C-R S$}

Here, we want to underline how an interpretation of a mathematical model (the graph) through GrC-RS methods can lead to new and more sophisticated tools in the granular computing field. We illustrate this aspect by introducing and discussing an original and natural extension of the classical concept of core. When we interpret various families of known graphs as information tables, also with quite different geometric structures, their 
core appears to be empty. Now, from an intuitive point of view, this contrasts with the different geometric nature of the considered graphs: for example, why a bipartite graph and a complete graph both have empty cores, although their geometric structures are significantly different? In order to give a valid answer to this question, we introduce a generalization of the core concept for any (not necessarily Boolean) information table J. An element of the extended core is now a set of attributes named essential (see Definition 4.1). We also show how the extended core is strictly related to the discernibility matrix (see Theorem 4.11).

Let us notice that the discernibility matrix of a simple graph $G$ uniquely characterizes $G$ in terms of a "local dissymmetry" (see Theorem 4.7) . This fact provides a specific geometric interpretation to any result concerning the simple graphs that can be obtained by means of GrC-RS methods.

Remark 1.1. In this paper we do not treat an important part of GrC-RS applied to the graph context. Specifically, we do not discuss rough lower and upper approximation functions, rough membership function and attribute dependency. For a detailed treatment concerning these notions in undirected graph theory we refer the reader to [8, 9, 11].

\subsection{Related works}

As anticipated, there already exist some works in GrC-GH, some of them also using rough sets (but differently as we do here). J. Stell is, to the best of our knowledge, the first to apply Granular Computing ideas to graphs. In [39] different kinds of granulation from a spatial standpoint are proposed: a partition of the nodes, a partition of the edges and of the nodes, a partition of the edges and a covering of the nodes. In some following works, he investigated the granularity for graphs and hypergraphs from the mathematical morphology and spatial information system perspectives [40, 41, 42].

The problem of defining granules on a graph is also discussed in [5], where a granule is a subset of vertices. Two methods are given to define a granule: one is based on the vertex degree of undirected graphs and the other on the distance among vertices of an undirected weighted graph. The same authors, in [6] introduce another possible way to define a granule as a set of edges instead of vertices.

The typical concept of level is used in [7] where hypergraphs are proposed as a model for granular structures: each hyperedge corresponds to a granule and different levels of granularity are represented by different hypergraphs; and in [2] where graph matching of labelled graphs is done using the $\mathrm{GrC}$ concepts of granulation and levels of abstraction.

Specific links between graph theory and rough set theory have been investigated in $[4,24,45,44,9,10]$. In [4] rough sets are used to test bipartiteness of simple undirected graphs. The representation of the graph is different from the one we are using here, indeed the relation at the basis of the rough-set investigation is a preclusive relation (as we also studied in [10]): two vertices are related iff they are connected.

In order to define lower an upper appproximations, a relation among edges (instead of vertices as we do) of a graph is considered in [24]. For instance, two labelled edges are equivalent if they have the same label. This approach is used to compute lower and upper bounds for the clique number of a graph.

Moving to more generalized rough sets, in [44] bipartite graphs are used to study covering-based rough sets: a representation of a covering through bipartite graph is 
given and then reducible elements of the covering are connected to 4-cycles of the graph.

We close this introductory section by briefly listing the contents of the paper. In section 2, we fix the notation used in the sequel, and we recall some basic facts concerning set partitions, rough sets and graphs. In this section, we also establish the first basic results concerning the interpretation of simple graphs as information tables. In section 3 we establish the main links between indiscernibility relations and graph automorphisms. Moreover we introduce some new topological concepts useful to interpret some properties of the geometric granulation on simple graphs. In section 4, we introduce the above discussed concept of extended core and we show that it is a useful extension of the classical core since in many cases the usual notion of core is not enough generic to differentiate among some distinct graph structures. We also establish two basic results concerning the extended core: one related to the discernibility matrix (Theorem 4.11) and another related to the reduct family (Theorem 4.20). In section 5, we apply the theoretical results of Section 4 to three graph families: the complete graph, the complete bipartite graph and the finite path on a fixed vertex number. Finally, in Section 6 we study in great detail the Petersen graph and we provide a purely combinatorial and geometric proof of the Theorem 6.6 on the structure of Petersen graph reducts and core.

\section{Notations, Recalls and First Results}

In this section, we firstly introduce the basic notions concerning the information tables that we will use in the paper. Next we establish the first basic relationships with graph theory. Table 1 summarizes our notation.

\begin{tabular}{|c|c|}
\hline Symbol & Meaning \\
\hline $\mathcal{P}(X)$ & the power set of a set $X$ \\
\hline$|X|$ & the cardinality of a finite set $X$ \\
\hline$\left(\begin{array}{l}X \\
k\end{array}\right)$ & the family of all the $k$-subsets of $X$ (i.e. the subsets of $X$ with \\
\hline & exactly $k$ elements), with $k$ a non-negative integer and $X$ a \\
\hline & finite set such that $|X| \geq k$ \\
\hline$\hat{n}$ & the set $\{1, \ldots, n\}$ with $n$ a positive integer \\
\hline $\begin{array}{c}v_{1}, \ldots, v_{n} \\
\text { (or } u_{1}, \ldots, u_{n} \text { ) }\end{array}$ & the vertices of a graph with a $n$-vertex set \\
\hline$I_{n}$ & the $n \times n$ identity matrix \\
\hline$J_{n}$ & the $n \times n$ matrix having 1 in all its entries \\
\hline$[x]_{R}$ & $\begin{array}{l}\text { the equivalence class of } x \text { with respect to the equivalence re- } \\
\text { lation } R\end{array}$ \\
\hline$\neg(x R y)$ & $\begin{array}{l}x \text { and } y \text { are not in relation with respect to the equivalence } \\
\text { relation } R\end{array}$ \\
\hline$A \triangle B$ & $\begin{array}{l}\text { the symmetric difference between } A \text { and } B \text {, that is } A \triangle B:= \\
(A \cup B) \backslash(A \cap B)\end{array}$ \\
\hline
\end{tabular}

Table 1: Explanation of the Notation

Let us notice that in some specific cases (for example in the case of the Petersen 
graph), in order to simplify the notation, we often write simply $1, \ldots, n$ instead of $v_{1}, \ldots, v_{n}\left(\right.$ or $\left.u_{1}, \ldots, u_{n}\right)$.

\subsection{Information Tables}

We start by recalling the notion of information table (see [28]) that plays a fundamental role in the scope of GrC-RS paradigm and in this paper.

Definition 2.1. An information table is a structure $\mathcal{J}=\langle U$, Att, Val, F $\rangle$, where $U=$ $\left\{u_{1}, u_{2}, \ldots, u_{m}\right\}$ is a nonempty finite set called universe set, Att $=\left\{a_{1}, a_{2} \ldots, a_{n}\right\}$ is a non empty finite set called the attribute set and $F: U \times A t t \rightarrow V a l$, called information map, is a function from the direct product $U \times A$ At into the value set $V$ al. The elements of $U$ are called objects and the elements of Att are called attributes. In particular, if $V a l=\{0,1\}$ we say that $\mathcal{J}$ is a Boolean information table.

The notion of information table has a relevant role in GrC-RS since many data come in tabular form and by virtue of a very natural equivalence relation that any attribute subset induces on the universe set. More specifically, if $A \subseteq A t t$, the $A$-indiscernibility relation $I_{A}$ on the universe set $U$ is defined as follows (see [28]): if $u, u^{\prime} \in U$ then

$$
u I_{A} u^{\prime}: \Longleftrightarrow F(u, a)=F\left(u^{\prime}, a\right), \forall a \in A
$$

If $u \in U$, we simply denote by $[u]_{A}$ (instead of $[u]_{I_{A}}$ ) the equivalence class of $u$ with respect to $I_{A}$. We also set

$$
\pi_{A}(\mathcal{J}):=\left\{[u]_{A}: u \in U\right\} .
$$

and call it the $A$-indiscernibility partition of the information table $\mathcal{J}$. In order to highlight that $\pi_{A}(\mathcal{J})$ is a set partition of the universe set $U$, we also use the notation

$$
\pi_{A}(\mathcal{J})=C_{1}|\ldots| C_{N}
$$

where $C_{i} \subseteq U$ are (all) the distinct equivalence classes $[u]_{A}$. We will also refer to the sets $C_{i}$ as the A-granules of $\mathcal{J}$.

\subsection{Simple Graphs as Information Tables}

For all general notions concerning undirected graphs we refer the reader to [13] and [18]. We always denote by $G=(V(G), E(G))$ a finite simple (i.e., without loops and multiple edges) undirected graph, with vertex set $V(G)=\left\{v_{1}, \ldots, v_{n}\right\}$ and edge set $E(G)$. If $v, v^{\prime} \in V(G)$, we will write $v \sim v^{\prime}$ if $\left\{v, v^{\prime}\right\} \in E(G)$ and $v \nsim v^{\prime}$ otherwise. If $v \in V(G)$, we set $N_{G}(v):=\{w \in V(G):\{v, w\} \in E(G)\}$, which is usually called the neighborhood of $v$ in $G$. As is well known, a graph can be defined through its adjacency matrix $\operatorname{Adj}(G)$ : the $n \times n$ matrix $\left(a_{i j}\right)$ such that $a_{i j}:=1$ if $v_{i} \sim v_{j}$ and $a_{i j}:=0$ otherwise.

In this paper we will study a graph $G$, and in particular its adjacency matrix, as if it were an information table. More formally:

Definition 2.2. We call information table of the graph $G$ the Boolean information table $\mathcal{J}[G]$, where the universe set and the attribute set of $\mathcal{J}[G]$ are both $\left\{v_{1}, \ldots, v_{n}\right\}$. The information map of $\mathcal{J}[G]$ is defined as $F\left(v_{i}, v_{j}\right):=1$ if $v_{i} \sim v_{j}$ and $F\left(v_{i}, v_{j}\right):=0$ otherwise. 
From now on, we write $\pi_{A}(G)$ instead of $\pi_{A}(\mathcal{J}[G])$, or simply $\pi_{A}$ if $G$ is clear from the context.

Definition 2.3. We call $\pi_{A}(G)$ the $A$-indiscernibility partition of the graph $G$. We use the term $A$-granule of $G$ for any block of the indiscernibility partition $\pi_{A}(G)$.

\subsection{Basics of Granular Computing on Simple Graphs}

With the term $G r C$ on Simple Graphs we mean the study of a new type of "geometry" on the graph $G$ having as its "points" exactly the A-granules of $G$, when A runs in the vertex subsets of $V(G)$. Since GrC-RS gives us several investigation tools in order to study such a type of relations between the $A$-granules of $G$, we naturally apply it to this new approach. The first elementary characterization of $\mathrm{GrC}$ on a generic simple graph $G$ is given by the following result.

Theorem 2.4. [8] Let $A \subseteq V(G)$ and $v, v^{\prime} \in V(G)$. The following conditions are equivalent:
(i) $v I_{A} v^{\prime}$.
(ii) For all $z \in A$ it results that $v \sim z$ if and only if $v^{\prime} \sim z$.
(iii) $N_{G}(v) \cap A=N_{G}\left(v^{\prime}\right) \cap A$.

As a direct consequence of the previous theorem, we obtain the general form of an $A$-granule for any simple graph $G$ :

Corollary 2.5. If $v \in V(G)$ and $A \subseteq V(G)$, then $[v]_{A}=\left\{v^{\prime}: N_{G}(v) \cap A=N_{G}\left(v^{\prime}\right) \cap A\right\}$.

Proof. It follows directly from the equivalence between $(i)$ and $($ iii) in Theorem 2.4.

The Theorem 2.4 also provides a sufficient condition for characterizing two vertices of the graph with no common edges.

Corollary 2.6. If $v I_{A} v^{\prime}$ and $\left\{v, v^{\prime}\right\} \cap A \neq \emptyset$, then $v \nsim v^{\prime}$.

Proof. It follows directly by Theorem 2.4 because there are no loops into $G$.

The result proved in Theorem 2.4 allows us to establish a basic link between the indiscernibility relation and its equivalent geometric characterization. In fact, once fixed a vertex subset $A$ of $G$, the geometric meaning of the conditions (ii) and (iii) of Theorem 2.4 is that an $A$-indiscernibility block consists of vertices having all the same incidence relation with respect to the vertices of $A$. According to Yao's terminology [49], it corresponds to a zooming-out operation on the vertex set $V(G)$ in order to reduce this set to only symmetric blocks with respect to $A$. Hence, from a geometric point of view, the choice of a fixed vertex subset $A$ can be thought of as the choice of a "symmetry axis" in the graph $G$ and the blocks of $\pi_{A}(G)$ as the "symmetric point subsets" with respect to $A$.

In the next definition we formalize the intuitive ideas just discussed.

Definition 2.7. If $\pi_{A}(G)$ is the partition generated by a fixed vertex subset $A$ of $G$, we call any equivalence class $B \in \pi_{A}(G)$ a symmetry block of $A$. The triple $\left(G, A, \pi_{A}(G)\right)$ is called $A$-granular reference system of $G$ or also granular reference system of $G$ with origin $A$. 
In Theorem 3.3 we will show that the terminology introduced in Definition 2.7 has a precise formal meaning in terms of graph automorphisms. This is a further justification to consider the $A$-granules of $G$ as symmetry blocks with respect to the origin $A$. We provide now an illustrative example of this idea on the Petersen graph.

Example 2.8. We consider the Petersen graph Pet of Figure 1. Let us fix the attribute subset $A=\{1,6\}$ and examine the $A$-granular reference system $\left(P e t, A, \pi_{A}(P e t)\right)$. In this case we have that $\pi_{A}=189|25| 347(10)$. This means that $A$ has three symmetry blocks : $\{1,8,9\},\{2,5,6\}$ and $\{3,4,7,10\}$. We notice that the vertices in a block have the same behavior with respect to the vertices 1 and 6 . In fact, 1,8 and 9 are the vertices of Pet that meet 6 but not 1 . The vertices 2,5 and 6 meet 1 but not 6 . The vertices 3 , 4, 7 and 10 do not meet 1 nor 6 . Finally, we note that there is no vertex that meets both 1 and 6 . We can see better the symmetric position of the previous blocks with respect to $A$ if we color them with three different colors:

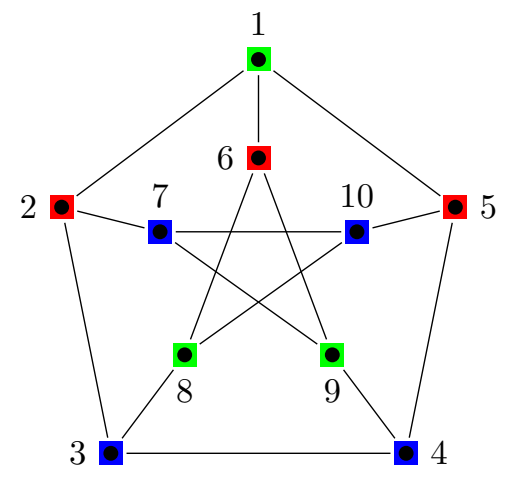

Figure 3: The blocks of the Petersen graph when $A=\{1,6\}$.

In the next example, we discuss the meaning of the symmetry blocks of a simple undirected graph derived from a real situation.

Example 2.9. Social network analysis is a research field which studies social structures through the use of networks or graphs. It characterizes networked structures in terms of vertices (things within the network) and the edges (interactions between the things in the network). A very used example (see for instance [14]) of such a network concerns the marriage ties of noble families in Florence in the 15th century. The vertices of the simple undirected graph $G$ in Figure 4 (adapted from [14]) represent the 16 elite Florentine families in the 15th century. Moreover, two vertices are connected with an edge if the two corresponding families are linked by a marriage tie. Then, if we take for example the vertex subset $A=\{9,13,16\}$, it is easy to verify that $\pi_{A}(\mathrm{G})=123(14)|4568(10)(11)(12)| 7|9|(13)|(15)|(16)$. In this real case, each block of $\pi_{A}(G)$ is a group of families having all a similar marriage relation with respect to the families in A: Medici, Ridolfi, Tornabuoni. Therefore, the vertices 1,2,3,14 are the families having a marriage relation with some member of the Medici family and no other marriage relation with members of the families Ridolfi or Tornabuoni. Analogously, the 
vertices $4,5,6,8,10,11,12$ are the families that have not marriage ties with no member of the families Medici, Ridolfi and Tornabuoni.

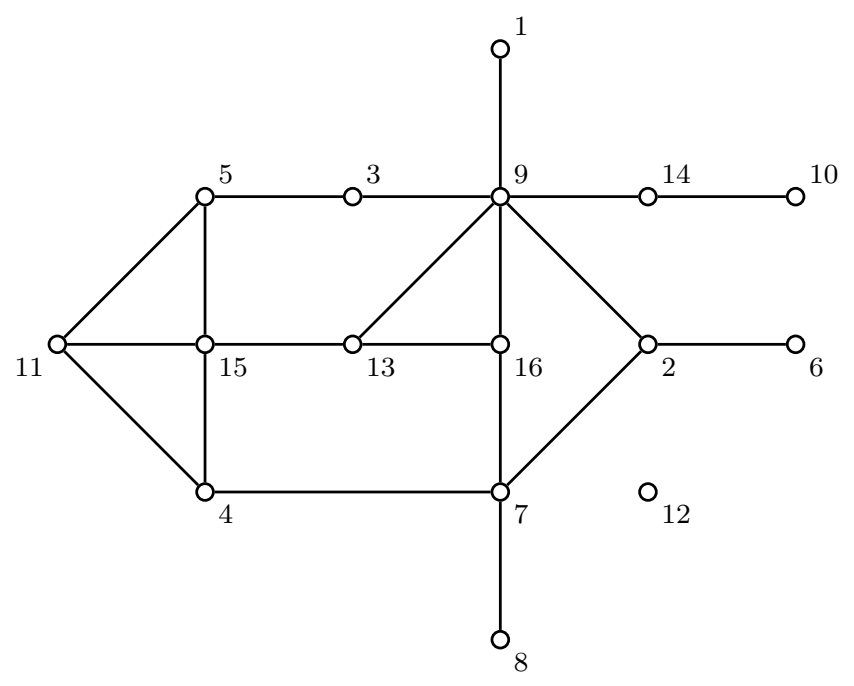
1. Acciaiuoli
9. Medici
2. Albizzi
10. Pazzi
3. Barbadori
11. Peruzzi
4. Bischeri
12. Pucci
5. Castellani
13. Ridolfi
6. Ginori
14. Salviati
7. Guadagni
15. Strozzi
8. Lamberteschi
16. Tornabuoni

Figure 4: The elite families in Florence in the 15th century

\subsection{Two Basic Cases: Complete and Bipartite Graphs}

A particularly simple case to be treated is the complete graph.

Definition 2.10. The complete graph on $n$ vertices, denoted by $K_{n}$, is the graph with vertex set $\left\{v_{1}, \ldots, v_{n}\right\}$ and such that $\left\{v_{i}, v_{j}\right\}$ is an edge, for any pair of indexes $i \neq j$.

We can easily determine the general form of any partition $\pi_{A}\left(K_{n}\right)$, for all $n \geq 1$ and all $A \subseteq V\left(K_{n}\right)$.

Proposition 2.11. [8] Let $n \geq 1$ and let $A=\left\{w_{1}, \ldots, w_{k}\right\}$ be a generic subset of $V\left(K_{n}\right)=\left\{v_{1}, \ldots, v_{n}\right\}$. Then

$$
\pi_{A}\left(K_{n}\right)=w_{1}\left|w_{2}\right| \ldots\left|w_{k}\right| A^{c},
$$

where $A^{c}$ is the complement set of $A$ in $V\left(K_{n}\right)$. 
We recall now the definition of bipartite graph.

Definition 2.12. A graph $B=(V(B), E(B))$ is said bipartite if there exist two nonempty subsets $B_{1}$ and $B_{2}$ of $V(b)$ such that $B_{1} \cap B_{2}=\emptyset, B_{1} \cup B_{2}=V(B)$ and $E(B) \subseteq$ $\left\{\{x, y\}: x \in B_{1}, y \in B_{2}\right\}$. In this case the pair $\left(B_{1}, B_{2}\right)$ is called a bipartition of $B$ and we write $B=\left(B_{1} \mid B_{2}\right)$. It is said that $B=\left(B_{1} \mid B_{2}\right)$ is a complete bipartite graph if $E(B)=\left\{\{x, y\}: x \in B_{1}, y \in B_{2}\right\}$. If $p$ and $q$ are two positive integers and $B_{1}=\left\{x_{1}, \ldots, x_{p}\right\}, B_{2}=\left\{y_{1}, \ldots, y_{q}\right\}$, we denote by $K_{p, q}$ the complete bipartite graph having bipartition $\left(B_{1}, B_{2}\right)$.

Also for $K_{p, q}$ we can easily describe its indiscernibility partitions.

Proposition 2.13. [8] Let $p$ and $q$ be two positive integers. Let $K_{p, q}=\left(B_{1} \mid B_{2}\right)$, where $B_{1}=\left\{x_{1}, \ldots, x_{p}\right\}$ and $B_{2}=\left\{y_{1}, \ldots, y_{q}\right\}$. Then $\pi_{A}\left(K_{p, q}\right)=x_{1} \ldots x_{p} \mid y_{1} \ldots y_{q}$ for each subset $A \subseteq V\left(K_{p, q}\right)$ such that $A \neq \emptyset$.

\subsection{Core and Reducts of Information Tables and Graphs}

In GrC-RS there are two particularly studied investigation tools: the core and the reducts of any fixed information table (see [28]). A reduct of an information table $\mathcal{J}$ is an attribute subset of $\mathcal{J}$ that provides the same knowledge of the whole attribute set and that is minimal with respect to this property. The core of $\mathcal{J}$ is the subset of all attributes of $\mathcal{J}$ whose elimination causes a substantial change in the knowledge induced from $\mathcal{J}$.

Definition 2.14. Let $\mathcal{J}=\langle U, A t t, V a l, F\rangle$ be a given information table. An attribute $c \in$ Att is said indispensable if $\pi_{A t t}(\mathcal{J}) \neq \pi_{A t t \backslash\{c\}}(\mathcal{J})$. The subset of all indispensable attributes of Att is called core of $\mathcal{J}$ and it is denoted by $C O R E(\mathcal{J})$. A subset $C \subseteq$ Att is said a reduct of $\mathcal{J}$ if:

(i) $\pi_{A t t}(\mathcal{J})=\pi_{C}(\mathcal{J})$;

(ii) $\pi_{\text {Att }}(\mathcal{J}) \neq \pi_{C \backslash\{c\}}(\mathcal{J})$ for all $c \in C$.

We denote by $R E D(\mathcal{J})$ the family of all reducts of $\mathcal{J}$.

Hence a reduct of $\mathcal{J}$ is an attribute subset that fully characterizes the knowledge in $\mathcal{J}$ and, moreover, it is also minimal with respect to this property: no attribute can be removed from this subset without causing a loss of knowledge. Core and reducts of $\mathcal{J}$ are linked by the following well-known result.

Proposition 2.15. [28] $C O R E(\mathcal{J}):=\bigcap\{C: C \in R E D(\mathcal{J})\}$.

We now report these notions on a simple undirected graph $G$.

Definition 2.16. We call core of $G$ the core of the information table $\mathcal{J}[G]$ and we denote it by $\operatorname{CORE}(G)$ instead of $\operatorname{CORE}(\mathcal{J}[G])$. We call core number of $G$, denoted by $\operatorname{Cnum}(G)$, the non negative integer $|C O R E(G)|$. Moreover we call reduct of $G$ any reduct of the information table $\mathcal{J}[G]$ and we denote by $R E D(G)$ the reduct family of $G$.

Remark 2.17. (i) Several notations depend on a fixed information table $\mathcal{J}$ or a graph $G$. From now on, when $\mathcal{J}$ or $G$ are clear from the context, we will omit the symbol $\mathcal{J}$ or $G$, respectively. For instance, we write simply $\pi_{A}$ instead of respectively $\pi_{A}(\mathcal{J})$ or $\pi_{A}(G)$. (ii) As we did in Definition 2.16, we often specialize a general concept concerning an information table to the information table associated to a graph $\mathcal{J}[G]$. In all these cases we use simply the letter $G$ instead of $\mathcal{J}[G]$ and we implicitly intend that the focused concept is automatically defined for the graph $G$, without further specifications. 


\section{General Geometric and Topological Properties}

We begin this section by showing that the $A$-indiscernibility relation in $G$ is closely related to the automorphism group of $G$. Then, a topological interpretation of the granular reference system of $G$ will be given.

\subsection{Graph Automorphisms and Indiscernibility Relation}

Firstly we recall the notion of automorphism of a graph.

Definition 3.1. Let $G=(V(G), E(G))$ be a graph (not necessarily a simple graph). An automorphism $\phi$ of $G$ is a bijective map $\phi: V(G) \rightarrow V(G)$ such that for all $x, y \in V(G)$ it results that

$$
\{x, y\} \in E(G) \Longleftrightarrow\{\phi(x), \phi(y)\} \in E(G) .
$$

The set of all the automorphisms of $G$ is a group with respect to the composition of maps and it is usually denoted by $\operatorname{Aut}(G)$.

In order to describe the $A$-indiscernibility in terms of induced subgraph automorphisms, we need to recall also the notion of induced subgraph of a graph.

Definition 3.2. Let $G=(V(G), E(G))$ be a simple graph and let $X$ be a fixed subset of $V(G)$. We call generated subgraph by $X$ in $G$, denoted by $G[X]$, the simple graph having $X$ as vertex set and such that if $x$ and $y$ are two distinct vertices in $X$, then $\{x, y\} \in E(G[X])$ if and only if $\{x, y\} \in E(G)$.

Now, let $A \subseteq V(G)$ be a fixed vertex subset of $G$ and let $v, v^{\prime}$ be two fixed vertices of $G$. Let $X:=A \cup\left\{v, v^{\prime}\right\}$. We define a map $\phi_{v, v^{\prime}}^{A}: X \rightarrow X$ as follows: if $x \in X$ we set

$$
\phi_{v, v^{\prime}}^{A}(x):= \begin{cases}x & \text { if } x \in A \backslash\left\{v, v^{\prime}\right\} \\ v^{\prime} & \text { if } x=v \\ v & \text { if } x=v^{\prime} .\end{cases}
$$

The next result shows that if $v \nsim v^{\prime}$ or $A \cap\left\{v, v^{\prime}\right\}=\emptyset$, then $v$ and $v^{\prime}$ are $A$-indiscernible if and only if $A$ is a type of "symmetry axis" with respect to $v$ and $v^{\prime}$. In other terms, the next result provides a mathematically precise meaning to the terminology introduced in Definition 2.7.

Theorem 3.3. Let $G=(V(G), E(G))$ be a simple graph, $A \subseteq V(G)$ and $v$, $v^{\prime}$ two distinct vertices of $G$. We set $X:=A \cup\left\{v, v^{\prime}\right\}$. Then, if $v \nsim v^{\prime}$ or $A \cap\left\{v, v^{\prime}\right\}=\emptyset$, we have that

$$
v I_{A} v^{\prime} \Longleftrightarrow \phi_{v, v^{\prime}}^{A} \in \operatorname{Aut}(G[X]) \text {. }
$$

Proof. We set $\psi:=\phi_{v, v^{\prime}}^{A}$. Firstly we assume that $v I_{A} v^{\prime}$. In order to prove that $\psi \in$ Aut $(G[X])$ we observe, by (4), that the map $\psi$ is bijective. Therefore we only need to show that for all $x, y \in X$ it results that

$$
x \sim y \Longleftrightarrow \psi(x) \sim \psi(y) .
$$

Let $x, y \in X$. Let us note that if $x, y \in A \backslash\left\{v, v^{\prime}\right\}$ by (4) we have $\psi(x)=x$ and $\psi(y)=y$, therefore (5) is satisfied. We can assume then that $\{x, y\} \cap\left\{v, v^{\prime}\right\} \neq \emptyset$. We distinguish two cases. Firstly, let us suppose that $x \in\left\{v, v^{\prime}\right\}$ and $y \notin\left\{v, v^{\prime}\right\}$ (the condition $x \notin\left\{v, v^{\prime}\right\}$ 
and $y \in\left\{v, v^{\prime}\right\}$ is similar. In this case we can assume without loss of generality that $x=v$. Since $y \in X$ and $y \notin\left\{v, v^{\prime}\right\}$, it follows that $y \in A$. By (ii) of Theorem 2.4 we obtain that

$$
v \sim y \Longleftrightarrow v^{\prime} \sim y .
$$

On the other hand, since $y \neq v$ and $y \neq v^{\prime}$, by (4) we deduce that $\psi(y)=y$, and this implies that (6) is equivalent to (5). The other case that we have to examine is $\{x, y\}=\left\{v, v^{\prime}\right\}$. We can assume without loss of generality that $x=v$ and $y=v^{\prime}$. By (4) it follows that $\psi(x)=y$ and $\psi(y)=x$, and due to the symmetry of the relation $\sim$ we obtain (5).

We suppose now that $\psi \in A u t(G[x])$ and we prove that for all $z \in A$ it results

$$
z \sim v \Longleftrightarrow z \sim v^{\prime} .
$$

By (ii) of Theorem 2.4, the equation (7) is equivalent to show that $v I_{A} v^{\prime}$. Let therefore $z \in A$. Firstly we assume that $z \notin\left\{v, v^{\prime}\right\}$. By (4) we have then $\psi(z)=z$. From this last identity and from the hypothesis $\psi \in \operatorname{Aut}(G[X])$ we deduce that

$$
v \sim z \Longleftrightarrow \psi(v) \sim \psi(z) \Longleftrightarrow v^{\prime} \sim z,
$$

that is exactly (7). Now, if $A \cap\left\{v, v^{\prime}\right\}=\emptyset$, since $z \in A$, it can not be $z \in\left\{v, v^{\prime}\right\}$. Hence, if $A \cap\left\{v, v^{\prime}\right\}=\emptyset$ the proof is completed. We can suppose then that $v \nsim v^{\prime}$ and $z \in\left\{v, v^{\prime}\right\}$. Without loss of generality we can assume that $z=v$. Since $G$ is a simple graph, $(z=v) \nsim v$, and by hypothesis $(z=v) \nsim v^{\prime}$, hence (7) is satisfied. This complete the proof.

The result proved in Theorem 3.3 is important since it clearly shows that the indiscernibility relation defined for a generic information table has a natural geometric interpretation in $G$ in terms of graph automorphisms.

The next result shows that the action of an automorphism of $G$ on any indiscernibility partition preserves its block partition structure.

Proposition 3.4. Let $G$ be a simple graph and let $\left(G, A, \pi_{A}(G)\right)$ be the granular reference system of $G$ with origin $A$. If $\phi \in \operatorname{Aut}(G)$ and $\pi_{A}(G)=B_{1}|\ldots| B_{N}$ then $\pi_{\phi(A)}(G)=\phi\left(B_{1}\right)|\ldots| \phi\left(B_{N}\right)$.

Proof. Firstly let us note that $\left\{\phi\left(B_{1}\right), \ldots, \phi\left(B_{N}\right)\right\}$ is a partition of $V(G)$ because the map $\phi$ is bijective and since $\left\{B_{1}, \ldots, B_{N}\right\}$ is a partition of $V(G)$. We set then $\pi:=$ $\phi\left(B_{1}\right)|\ldots| \phi\left(B_{N}\right)$ and $\pi_{\phi(A)}(G)=C_{1}|\ldots| C_{M}$. In order to obtain the thesis we must prove that

$$
\phi\left(B_{1}\right)|\ldots| \phi\left(B_{N}\right)=C_{1}|\ldots| C_{M} .
$$

Since $\pi$ and $\pi_{\phi(A)}(G)$ are both partitions of $V(G)$, it is clear that the proof of (8) is equivalent to showing that

$$
\forall C_{j} \exists B_{i}: C_{j}=\phi\left(B_{i}\right) .
$$

Let therefore $C_{j}$ be a generic block of $\pi_{\phi(A)}(G)$ and let $u$ be a fixed element of $C_{j}$. So that we have $C_{j}=[u]_{\phi(A)}$. Let $w \in V(G)$ such that $\phi(w)=u$. Since $\left\{B_{1}, \ldots, B_{N}\right\}$ is a partition of $V(G)$, there exists a block $B_{i}$ such that $w \in B_{i}$. In order to obtain (9), we will prove that

$$
[u]_{\phi(A)}=\phi\left(B_{i}\right) .
$$


For all $u^{\prime} \in V(G)$, by (ii) of Theorem 2.4 we have that

$$
u I_{\phi(A)} u^{\prime} \Longleftrightarrow\left((\forall z \in \phi(A)): u \sim z \Longleftrightarrow u^{\prime} \sim z\right) .
$$

Since $\phi$ is an automorphism of $G$, the condition (11) is equivalent to the following:

$$
u I_{\phi(A)} u^{\prime} \Longleftrightarrow\left((\forall y \in A): \phi^{-1}(u) \sim y \Longleftrightarrow \phi^{-1}\left(u^{\prime}\right) \sim y\right) .
$$

Therefore, again by (ii) of Theorem 2.4 we obtain:

$$
u I_{\phi(A)} u^{\prime} \Longleftrightarrow \phi^{-1}(u) I_{A} \phi^{-1}\left(u^{\prime}\right) .
$$

Now, let $u^{\prime} \in[u]_{\phi(A)}$ and $w^{\prime} \in V(G)$ such that $\phi\left(w^{\prime}\right)=u^{\prime}$. Since $u I_{\phi(A)} u^{\prime}$, by (13) we deduce that $w=\phi^{-1}(u) I_{A} \phi^{-1}\left(u^{\prime}\right)=w^{\prime}$. But $w \in B_{i}$ and $B_{i}$ is an indiscernibility block of the equivalence relation $I_{A}$, therefore we also deduce by (13) that $w^{\prime} \in B_{i}$. Hence $u^{\prime}=\phi\left(w^{\prime}\right) \in \phi\left(B_{i}\right)$, and this shows that $[u]_{\phi(A)} \subseteq \phi\left(B_{i}\right)$.

On the other hand, let $u^{\prime} \in \phi\left(B_{i}\right)$. Then there exists $w^{\prime} \in B_{i}$ such that $\phi\left(w^{\prime}\right)=u^{\prime}$. Since $w, w^{\prime} \in B_{i}$ and $B_{i}$ is an indiscernibility block of $I_{A}$, we have that $\phi^{-1}(u)=w I_{A} \phi^{-1}\left(u^{\prime}\right)=$ $w^{\prime}$ and, by (13), this implies $u I_{\phi(A)} u^{\prime}$, i.e. $u^{\prime} \in[u]_{\phi(A)}$. This shows that $\phi\left(B_{i}\right) \subseteq[u]_{\phi(A)}$. Hence (10) is proved.

In the next result, we show that the automorphisms of $G$ preserve its reducts.

Proposition 3.5. Let $G$ be a simple undirected graph. If $A \subseteq V(G)$ and $\phi$ is an automorphism of $G$ then

$$
A \in R E D(G) \Longleftrightarrow \phi(A) \in R E D(G)
$$

Proof. Let $A \in R E D(G)$. By Proposition 3.4, if $\pi_{A}(G)=B_{1}|\ldots| B_{N}$, then $\pi_{\phi(A)}(G)=$ $\phi\left(B_{1}\right)|\ldots| \phi\left(B_{N}\right)$. Let $u, v \in V(G)$ such that $u I_{\phi(A)} v$. Then $u, v \in \phi\left(B_{i}\right)$ for some $i \in$ $\{1, \ldots, N\}$. This is equivalent to say that $\phi^{-1}(u), \phi^{-1}(v) \in B_{i}$ for some $i \in\{1, \ldots, N\}$, namely $\phi^{-1}(u) I_{A} \phi^{-1}(v)$. Since $A$ is a reduct of $G$, we have $\phi^{-1}(u) I_{A t t} \phi^{-1}(v)$. Thus, since $A t t=\phi^{-1}(A t t), \phi^{-1}(u) I_{\phi^{-1}(A t t)} \phi^{-1}(v)$. Therefore for each $a \in A t t, \phi^{-1}(u) \| \phi^{-1}(a)$ if and only if $\phi^{-1}(v) \| \phi^{-1}(a)$. Finally, $\phi \in \operatorname{Aut}(G)$ implies $u \| a$ if and only if $v \| a$, so $u I_{A t t} v$. This proves that $\pi_{\phi(A)}(G)=\pi_{A t t}(G)$.

Now let $a \in A$. Since $A$ is a reduct $\pi_{A \backslash\{a\}}(G) \neq \pi_{A t t}(G)$. Thus there exist $u, v \in A t t$ such that $u I_{A \backslash\{a\}} v$ and $\neg\left(u I_{A t t} v\right)$. By Proposition 3.4 we have $\phi(u) I_{\phi(A) \backslash\{\phi(a)\}} \phi(v)$, but at the same time $\neg\left(\phi(u) I_{A t t} \phi(v)\right)$. Thus $\pi_{\phi(A) \backslash\{\phi(a)\}}(G) \neq \pi_{A t t}(G)$ and so $\phi(A) \in$ $R E D(G)$.

The other implication holds too because $\phi^{-1}$ is also an automorphism.

\subsection{Topological properties}

Now, we show that the study of the granular reference system of $G$ with a fixed origin $A$ is closely connected with some "topological type" properties of the vertex subset $A$. In order to delineate such a type of "topology" we introduce the following terminology.

Definition 3.6. Let $A$ be a vertex subset of $G$ and let $v \in V(G)$. We say then that:

(i) $v$ is $A$-isolated if $v \in A$ and $N_{G}(v) \subseteq V(G) \backslash A$;

(ii) $v$ is $A$-inner if $v \in A$ and $N_{G}(v) \subseteq A$;

(iii) $v$ is $A$-outer if $v \notin A$ and $N_{G}(v) \subseteq V(G) \backslash A$; 
(iv) $v$ is $A$-delimiting if $N_{G}(v) \cap A \neq \emptyset$ and $N_{G}(v) \cap V(G) \backslash A \neq \emptyset$;

(v) $v$ is $A$-surrounded if $v \notin A$ and $N_{G}(v) \subseteq A$.

We denote respectively by $\operatorname{Iso}(A), \operatorname{Inn}(A), \operatorname{Out}(A), \operatorname{Del}(A)$ and $\operatorname{Sur}(A)$ the subsets of all A-isolated, A-inner, A-outer, A-delimiting and A-surrounded vertices of $G$. It is clear that the family of all previous subsets is a set partition of $V(G)$, that we denote by $\tau_{A}(G)$ (or simply by $\tau_{A}$ if $G$ is clear from the context). We call $\tau_{A}(G)$ the A-topological partition of $G$.

Proposition 3.7. Let $A \subseteq V(G)$ and $A^{c}:=V(G) \backslash A$. Then $\operatorname{Iso}(A)=\operatorname{Sur}\left(A^{c}\right)$, $\operatorname{Inn}(A)=\operatorname{Out}\left(A^{c}\right)$ and $\operatorname{Del}(A)=\operatorname{Del}\left(A^{c}\right)$.

Proof. It follows directly by the previous definitions.

We have then the following result which relates $\tau_{A}(G)$ with $\pi_{A}(G)$.

Proposition 3.8. Let $A \subseteq V(G)$. Then:

(i) If $\operatorname{Iso}(A) \cup \operatorname{Out}(A) \neq \emptyset$ then the subset $\operatorname{Iso}(A) \cup \operatorname{Out}(A)$ is a block of the $A$ indiscernibility partition $\pi_{A}(G)$.

(ii) If $v, v^{\prime} \in \operatorname{Inn}(A) \cup \operatorname{Sur}(A)$ then

$$
v I_{A} v^{\prime} \Longleftrightarrow N_{G}(v)=N_{G}\left(v^{\prime}\right) .
$$

Proof. (i) Let $v, v^{\prime} \in I \operatorname{so}(A) \cup \operatorname{Out}(A)$. Then, for each $z \in A$, we have $F(z, v)=$ $F\left(z, v^{\prime}\right)=0$, so $v I_{A} v^{\prime}$. Thus $I s o(A) \cup \operatorname{Out}(A)$ contains a block of $\pi_{A}(G)$. Let now $u \in V(G)$ such that, for some $v \in I \operatorname{so}(A) \cup \operatorname{Out}(A), u I_{A} v$. Then $N_{G}(u) \cap A=\emptyset$. In fact, if (by absurd) there exists a vertex $z$ in $N_{G}(u) \cap A$, then $1=F(z, u)=F(z, v)=0$ and this contradicts the assumption that $u I_{A} v$. Then $N_{G}(u) \cap A=\emptyset$ and thus $u$ is either a $A$-isolated or a $A$-outer vertex.

(ii) Let $v, v^{\prime} \in \operatorname{Inn}(\mathrm{A}) \cup \operatorname{Sur}(A)$. If $N_{G}(v)=N_{G}\left(v^{\prime}\right)$, then for each $z \in A, F(z, v)=$ $F\left(z, v^{\prime}\right)=1$ if $z \in N_{G}(v)$ and $F(z, v)=F\left(z, v^{\prime}\right)=0$ if $z \notin N_{G}(v)$, so $v I_{A} v^{\prime}$. Let us suppose now $N_{G}(v) \neq N_{G}\left(v^{\prime}\right)$ and let $z \in N_{G}(v) \triangle N_{G}\left(v^{\prime}\right)$. Since both $N_{G}(v)$ and $N_{G}\left(v^{\prime}\right)$ are contained in $A, z \in A$ and $F(z, v) \neq F\left(z, v^{\prime}\right)$. This implies $\neg\left(v I_{A} v^{\prime}\right)$.

Corollary 3.9. Let $A \subseteq V(G)$. Then:

(i) If $\operatorname{Sur}(A) \cup \operatorname{Inn}(A) \neq \emptyset$ then the subset $\operatorname{Sur}(A) \cup \operatorname{Inn}(A)$ is a block of the $A^{c}$ indiscernibility partition $\pi_{A^{c}}(G)$.

(ii) If $v, v^{\prime} \in \operatorname{Out}(A) \cup I \operatorname{so}(A)$ then

$$
v I_{A^{c}} v^{\prime} \Longleftrightarrow N_{G}(v)=N_{G}\left(v^{\prime}\right) .
$$

Proof. Both parts (i) and (ii) follow directly by Propositions 3.7 and 3.8.

When $G=K_{n}$ is the complete graph, we have the following result.

Proposition 3.10. Let $A \subseteq V\left(K_{n}\right)$, with $n>2$. Then:

(i) If $|A|=1$, then $\operatorname{Del}(A)=V\left(K_{n}\right) \backslash A$ and Iso $(A)=A$.

(ii) If $2 \leq|A| \leq n-2$, then $\operatorname{Del}(A)=V\left(K_{n}\right)$.

(iii) If $|\bar{A}|=n-1$, then $\operatorname{Del}(A)=A$ and $\operatorname{Sur}(A)=V\left(K_{n}\right) \backslash A$.

(iv) $\operatorname{Inn}\left(K_{n}\right)=\operatorname{Out}(\emptyset)=V\left(K_{n}\right)$. 
Proof. (i) If $A=\{u\}$, with $u \in V\left(K_{n}\right)$, then $N_{K_{n}}(u)=V\left(K_{n}\right) \backslash A$, so $u \in I \operatorname{so}(A)$. Let $v \in V\left(K_{n}\right) \backslash A$. In this case, for each $v \in V\left(K_{n}\right)$, we have $u \in N_{K_{n}}(v)$ and $N_{K_{n}}(v) \cap\left(V\left(K_{n}\right) \backslash\{u\}\right) \neq \emptyset$. Thus $v \in \operatorname{Del}(A)$.

(ii) If $2 \leq|A| \leq n-2$, then, for each $v \in V\left(K_{n}\right), N_{K_{n}}(v) \cap A \neq \emptyset$ and $N_{K_{n}}(v) \cap\left(V\left(K_{n}\right) \backslash\right.$ $A) \neq \emptyset$. Thus $\operatorname{Del}(A)=V\left(K_{n}\right)$.

(iii) The thesis follows by part (i) and by Proposition 3.7.

(iv) It is trivial and it follows directly from the previous definitions.

Analogously, when $G=K_{p, q}$ is the complete bipartite graph, we obtain:

Proposition 3.11. Let $A \subseteq V\left(K_{p, q}\right)$, with bipartition $\left(B_{1} \mid B_{2}\right)$. Then:

(i) if $A=V\left(K_{p, q}\right)$ we have $\operatorname{Inn}(A)=V\left(K_{p, q}\right)$;

(ii) if $B_{1} \subseteq A \varsubsetneqq V\left(K_{p, q}\right)$ we have $\operatorname{Inn}(A)=B_{2} \cap A$, Sur $(A)=V\left(K_{p, q}\right) \backslash A$, Out $(A)=\emptyset$,

$$
\operatorname{Del}(A)= \begin{cases}B_{1} & \text { if } B_{1} \varsubsetneqq A \\ \emptyset & \text { if } B_{1}=A,\end{cases}
$$

and

$$
I s o(A)= \begin{cases}\emptyset & \text { if } B_{1} \varsubsetneqq A, \\ A & \text { if } B_{1}=A .\end{cases}
$$

Analogously if $B_{2} \subseteq A$

(iii) If $A \neq \emptyset, B_{1} \nsubseteq A$ and $B_{2} \nsubseteq A$ we have that $\operatorname{Del}(A)=V\left(K_{p, q}\right)$.

Proof. (i) It holds for any graph $G$.

(ii) Let $A \subseteq B_{1}$, with $A \neq V\left(K_{p, q}\right)$. If $v \in B_{2} \cap A$, then $N_{K_{p, q}}(v) \cap A=B_{1} \subseteq A$ and thus $v \in \operatorname{Inn}(A)$. On the other hand, if $v \in B_{1}$ then $N_{K_{p, q}}(v)=B_{2}$, thus $N_{K_{p, q}}(v) \cap A=B_{2} \cap A$ and $N_{K_{p, q}}(v) \cap\left(V\left(K_{p, q}\right) \backslash A\right)=V\left(K_{p, q}\right) \backslash A$. It follows that $\operatorname{Inn}(A)=B_{2} \cap A$ and

$$
I s o(A)= \begin{cases}\emptyset & \text { if } B_{1} \varsubsetneqq A, \\ A & \text { if } B_{1}=A .\end{cases}
$$

Let $v \in V\left(K_{p, q}\right) \backslash A$. Then $N_{K_{p, q}}(v)=B_{1} \subseteq A$, so $\operatorname{Sur}(A)=V\left(K_{p, q}\right) \backslash A$, Out $(A)=\emptyset$ and

$$
\operatorname{Del}(A)= \begin{cases}B_{1} & \text { if } B_{1} \varsubsetneqq A \\ \emptyset & \text { if } B_{1}=A .\end{cases}
$$

(iii) Let $A \neq \emptyset$ such that $B_{1} \nsubseteq A$ and $B_{2} \nsubseteq \nsubseteq A$. If $v \in V\left(K_{p, q}\right)$, then $N_{K_{p, q}}(v) \cap A \neq \emptyset$ and $N_{K_{p, q}}(v) \cap V\left(K_{p, q}\right) \backslash A \neq \emptyset$. This completes the proof.

\section{Extended Core and Discernibility Hypergraph of an Information Table}

In this section we introduce an extended notion of core, which is useful in better characterizing a graph than the standard core. Moreover, we relate this new notion to the problem of finding all the reducts (of a generic information table) through the minimal hypergraph transversal problem. 
In agreement with Remark 2.17, all the notions that we will introduce in this section for a generic information table must be understood also valid for any simple graph $G$ (in this case we use only the letter $G$ instead of the symbol $\mathcal{J}[G]$ ).

\subsection{The Extended Core}

We generalize the concept of classical Pawlak core for a generic information table $\mathcal{J}$. This step is needed since in many situations the simple core of a graph is empty, see for instance the cases of complete and bipartite complete graphs in Corollary 5.3.

Definition 4.1. We say that a subset $C \subseteq$ Att is J-essential (or, simply, essential) if:

(i) $\pi_{A t t \backslash C}(\mathcal{J}) \neq \pi_{A t t}(\mathcal{J})$;

(ii) for all $D \varsubsetneqq C$ we have that $\pi_{A t t \backslash D}(\mathcal{J})=\pi_{A t t}(\mathcal{J})$.

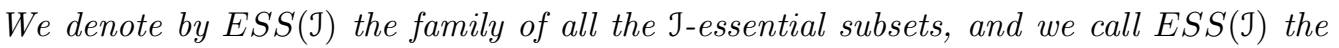
extended core of $\mathcal{J}$. If $k \in\{1, \ldots, n\}$, we set

$$
\operatorname{ESS}_{k}(\mathcal{J}):=\{C \in \operatorname{ESS}(\mathcal{J}):|C|=k\}
$$

and we call essential numerical sequence of $\mathcal{J}$ the $n$-tuple

$$
\operatorname{ens}(\mathcal{J}):=\left(\left|E S S_{1}(\mathcal{J})\right|, \ldots,\left|E S S_{n}(\mathcal{J})\right|\right) .
$$

Finally, we call essential dimension of $\mathcal{J}$ the positive integer

$$
\operatorname{Edim}(\mathcal{J}):=\min \left\{k:\left|E S S_{k}(\mathcal{J})\right| \neq 0\right\} .
$$

The following result shows that the subset family $\operatorname{ESS}(\mathcal{J})$ is effectively an extension of the classical core of $\mathcal{J}$.

Proposition 4.2. $\operatorname{CORE}(\mathcal{J})=\bigcup\left\{C: C \in E S S_{1}(\mathcal{J})\right\}$, therefore $|C O R E(\mathcal{J})|=\left|E S S_{1}(\mathcal{J})\right|$.

Proof. It follows immediately from the definitions of $\operatorname{CORE}(\mathcal{J})$ and $E S S_{1}(\mathcal{J})$.

In particular, for any simple graph $G$ we have that $\operatorname{Cnum}(G)=\left|E S S_{1}(G)\right|$.

By virtue of Proposition 4.2 we can always identify $C O R E(\mathcal{J})$ with the singleton family $E S S_{1}(\mathcal{J})$. Therefore it is natural to think of the classical Pawlak core as a type of "first level core" for the information table $\mathcal{J}$. This leads naturally to the following definition.

Definition 4.3. With the previous notation, if $1 \leq k \leq n$, we call $k$-th core of the information table $\mathcal{J}$ the subset family $E S S_{k}(\mathcal{J})$.

The discernibility matrix of $\mathcal{J}[36]$ is an essential tool to determine core and reducts of $\mathcal{J}$.

Definition 4.4. Let $\mathcal{J}=\langle U, A t t, V a l, F\rangle$ an information table such that $|U|=m$ and $U=\left\{u_{1}, \ldots, u_{m}\right\}$. The discernibility matrix $\Delta[\mathcal{J}]$ of $\mathcal{J}$ is the $m \times m$ matrix $\left(d_{i j}\right)$, where

$$
d_{i j}=\left\{a \in A t t: F\left(u_{i}, a\right) \neq F\left(u_{j}, a\right)\right\}:=\Delta_{\mathcal{J}}\left(u_{i}, u_{j}\right) .
$$


Therefore the entry $d_{i j}=\Delta_{\mathcal{J}}\left(u_{i}, u_{j}\right)$ is the subset of all attributes $a \in$ Att which discern the object $u_{i}$ from the object $u_{j}$. Let us note that $\Delta[\mathcal{J}]$ is a symmetric matrix such that $\Delta_{\mathcal{J}}\left(u_{i}, u_{i}\right)=\emptyset$ for all $i=1, \ldots, m$.

It is also convenient to introduce a numerical version of the discernibility matrix.

Definition 4.5. The numerical discernibility matrix $\Delta_{\text {num }}[\mathcal{J}]$ of $\mathcal{J}$ is the $m \times m$ matrix $\left(\delta_{i j}\right)$, where $\delta_{i j}:=\left|\Delta_{\mathcal{J}}\left(u_{i}, u_{j}\right)\right|$.

In the next result we describe the discernibility matrix $\Delta[G]$ for an arbitrary simple graph $G$ in geometrical terms.

Proposition 4.6. If $G$ is a simple undirected graph and $u_{i}, u_{j} \in V(G)$, then

$$
\Delta_{G}\left(u_{i}, u_{j}\right)=N_{G}\left(u_{i}\right) \triangle N_{G}\left(u_{j}\right) .
$$

Proof. By (17) and by definition of indiscernibility relation we have that

$$
\Delta_{G}\left(u_{i}, u_{j}\right)=\left\{v \in V(G): \neg\left(u_{i} I_{\{v\}} u_{j}\right)\right\} .
$$

By (ii) of Theorem 2.4 and (19) we deduce that

$$
\Delta_{G}\left(u_{i}, u_{j}\right)=\left\{v \in V(G): \neg\left(u_{i} \sim v \Longleftrightarrow u_{j} \sim v\right)\right\},
$$

which, in terms of $N_{G}\left(u_{i}\right)$ and $N_{G}\left(u_{j}\right)$, is equivalent to

$$
\Delta_{G}\left(u_{i}, u_{j}\right)=\left\{v \in V(G): \neg\left(v \in N_{G}\left(u_{i}\right) \Longleftrightarrow v \in N_{G}\left(u_{j}\right)\right\} .\right.
$$

By (20) we deduce then that

$$
\Delta_{G}\left(u_{i}, u_{j}\right)=\left\{v \in V(G):\left(v \in N_{G}\left(u_{i}\right) \wedge v \notin N_{G}\left(u_{j}\right)\right) \vee\left(v \in N_{G}\left(u_{j}\right) \wedge v \notin N_{G}\left(u_{i}\right)\right)\right\} .
$$

We observe now that (21) is exactly the thesis.

To provide a geometrical interpretation of the identity (18), we can observe that if $u_{i} \neq u_{j}$ then $v \in \Delta_{G}\left(u_{i}, u_{j}\right)$ iff $v$ is connected with exactly one vertex between $u_{i}$ and $u_{j}$; this means that we can think $v$ as a dissymmetry vertex for $u_{i}$ and $u_{j}$. Therefore it is natural also to call $\Delta_{G}\left(u_{i}, u_{j}\right)$ the local dissymmetry axis of $u_{i}$ and $u_{j}$ and local dissymmetry number of $u_{i}$ and $u_{j}$ the integer $\delta_{i j}:=\left|\Delta_{G}\left(u_{i}, u_{j}\right)\right|$. In the next result we show that the local dissymmetry uniquely characterizes a finite simple graph having a fixed vertex set $V$.

Theorem 4.7. Let $G_{1}$ and $G_{2}$ be two finite simple graphs such that $V=V\left(G_{1}\right)=V\left(G_{2}\right)$. Then

$$
\Delta\left[G_{1}\right]=\Delta\left[G_{2}\right] \Longleftrightarrow G_{1}=G_{2} .
$$

Proof. Suppose that $\Delta_{G_{1}}\left(v_{i}, v_{j}\right)=\Delta_{G_{2}}\left(v_{i}, v_{j}\right)$ for every $v_{i}, v_{j}$, then $F_{G_{1}}(v, w)=F_{G_{2}}(v, w)$ for every pair of vertices $v$ and $w$, because in $G$ there are no loops. Then it follows immediately that $E\left(G_{1}\right)=E\left(G_{2}\right)$, hence $G_{1}=G_{2}$. 
The result established in Theorem 4.7 tells us that the discernibility matrix of a finite simple undirected graph $G$ uniquely characterizes $G$, therefore it has the same theoretical relevance of the adjacency matrix of $G$ (when $G$ is simple). Now, since the discernibility matrix can be considered in GrC-RS as the fundamental tool to determine core and reducts of an information system, all the possible investigations concerning the simple graph $G$ by means of GrC-RS tools must necessarily conduct to a well specific characterization of $G$ in terms of local dissymmetry.

In [36] it has been proved that for any information table $\mathcal{J}=\langle U, A t t, V, F\rangle$, with $U=\left\{u_{1}, \ldots, u_{m}\right\}$, the core of $\mathcal{J}$ coincides with the set of all single element entries of the discernibility matrix of $\mathcal{J}$, i.e.

$$
\operatorname{CORE}(\mathcal{J})=\left\{a \in A t t: \exists i, j: \Delta_{\mathcal{J}}\left(u_{i}, u_{j}\right)=\{a\}\right\} .
$$

If $D I S C(\mathcal{J})$ is the family of all the distinct non-empty entries $\Delta_{\mathcal{J}}\left(u_{i}, u_{j}\right)$ of the discernibility matrix $\Delta[\mathcal{J}]$, then $(22)$ is obviously equivalent to say that for all $a \in A t t$ :

$$
a \in C O R E(\mathcal{J}) \Longleftrightarrow\{a\} \in D I S C(\mathcal{J}) .
$$

Given this result, we obtain a completely geometric characterization of the core of a graph.

Proposition 4.8. $\operatorname{CORE}(G)=\left\{v \in V(G): \exists x, y \in V(G): N_{G}(x) \triangle N_{G}(y)=\{v\}\right\}$.

Proof. The universe set and the attribute set of the information table $\mathcal{J}[G]$ are both (by definition of $\mathcal{J}[G]$ ) the vertex set $V(G)$, hence, by $(22)$, we have that

$$
\operatorname{CORE}(G)=\left\{v \in V(G): \exists x, y \in V(G): \Delta_{G}(x, y)=\{v\}\right\} .
$$

The thesis follows then directly from (18) and (24).

Corollary 4.9. If $u_{1}, \ldots, u_{m}$ are the distinct vertices of $G$, then

$$
\operatorname{Cnum}(G)=0 \Longleftrightarrow \delta_{i j} \neq 1 \forall i \neq j
$$

Proof. The thesis follows by (18) and Proposition 4.8 because $\operatorname{Cnum}(G):=|\operatorname{CORE}(G)|$ and $\delta_{i j}:=\left|\Delta_{G}\left(u_{i}, u_{j}\right)\right|=\left|N_{G}\left(u_{i}\right) \triangle N_{G}\left(u_{j}\right)\right|$.

In order to describe all the elements of $\operatorname{ESS}(\mathcal{J})$, we introduce the discernibility poset.

Definition 4.10. We call discernibility poset of the information table $\mathcal{J}$, the poset

$$
\mathbb{D}(\mathcal{J}):=(D I S C(\mathcal{J}), \subseteq)
$$

which is a sub-poset of the power set lattice $(\mathcal{P}(A t t), \subseteq)$.

In the next result we establish a basic link between essential subsets and discernibility subsets.

Theorem 4.11. ESS (J) coincides with the family of all the minimal elements in the poset $\mathbb{D}(\mathcal{J})$. 
Proof. Let $C \in E S S(\mathcal{J})$. Since $\pi_{A t t \backslash C}(\mathcal{J}) \neq \pi_{A t t}(\mathcal{J})$, there exist (at least) two distinct elements $u, u^{\prime} \in U$ such that $u I_{A t t \backslash C} u^{\prime}$ and $\neg\left(u I_{A t t} u^{\prime}\right)$. Therefore, since $F(u, a)=$ $F\left(u^{\prime}, a\right)$ for all $a \in A t t \backslash C$, by (17) we deduce that Att $\backslash C \subseteq A t t \backslash \Delta_{\mathcal{J}}\left(u, u^{\prime}\right)$, i.e. $C \supseteq \Delta_{\mathcal{J}}\left(u, u^{\prime}\right)$. We prove now that also the reverse inclusion is true. Let us assume, by contradiction, that there exists an attribute $c \in C$ such that $c \notin \Delta_{\mathcal{J}}\left(u, u^{\prime}\right)$, so that by (17) we have $F(u, c)=F\left(u^{\prime}, c\right)$. Let $D:=C \backslash\{c\}$. From the conditions $F(u, a)=F\left(u^{\prime}, a\right)$ for all $a \in A t t \backslash C$ and $F(u, c)=F\left(u^{\prime}, c\right)$ we deduce that $F(u, a)=F\left(u^{\prime} a\right)$ for all $a \in A t t \backslash C \cup\{c\}=A t t \backslash D$, that is equivalent to $u I_{A t t \backslash D} u^{\prime}$. Then, since $\neg\left(u I_{A t t} u^{\prime}\right)$ we obtain that $\pi_{A t t \backslash D}(\mathcal{J}) \neq \pi_{A t t}(\mathcal{J})$, and this is in contrast with (ii) of Definition 4.1. Then we also have $C \subseteq \Delta_{\mathcal{J}}\left(u, u^{\prime}\right)$, therefore $C=\Delta_{\mathcal{J}}\left(u, u^{\prime}\right)$. This shows that $C \in D I S C(\mathcal{J})$. We must prove now that $C$ is minimal in $D I S C(\mathcal{J})$ with respect to the inclusion. Let us assume (by contradiction) that there exists some $D \in D I S C(\mathcal{J})$ such that $D \varsubsetneqq C$. By definition of $D I S C(\mathcal{J})$ we have that $D=\Delta_{\mathcal{J}}(v, w)$, for some $v, w \in U$ with $u \neq w$. Then Att $\backslash D=\{a \in A t t: F(u, a)=F(v, a)\}$, and this is equivalent to say that $v I_{A t t \backslash D} w$. On the other hand, since $D \neq \emptyset$ we can choose an element $a_{0} \in D$, and this implies that $F\left(v, a_{0}\right) \neq F\left(w, a_{0}\right)$ because $D=\Delta_{\mathcal{J}}(v, w)$. This shows that $\neg\left(v I_{A t t} w\right)$. Then from the conditions $v I_{A t t \backslash D} w$ and $\neg\left(v I_{A t t} w\right)$ we deduce that $\pi_{A t t \backslash D}(\mathcal{J}) \neq \pi_{A t t}(\mathcal{J})$, that is in contrast with the hypotheses that $C$ is an essential subset for $\mathcal{J}$ and $D \varsubsetneqq C$. This proves that $C$ is minimal. Hence each element $C \in E S S(\mathcal{J})$ is a minimal element of the poset $\mathbb{D}(\mathcal{J})$. In order to complete the proof it remains to show that each minimal element of the poset $\mathbb{D}(\mathcal{J})$ is an essential subset. Let therefore $C \in D I S C(\mathcal{J})$ which is also minimal with respect to the inclusion. We must prove that $C \in \operatorname{ESS}(\mathcal{J})$. By definition of $D I S C(\mathcal{J})$, there are two distinct elements $u, u^{\prime} \in U$ such that $C=\Delta_{\mathcal{J}}\left(u, u^{\prime}\right)=\left\{a \in\right.$ Att $\left.: F(u, a) \neq F\left(u^{\prime}, a\right)\right\}$. Since $C \neq \emptyset$ (by definition of $\operatorname{DISC}(\mathcal{J})$ ), we choose a fixed element $c \in C$, so that $F(u, c) \neq F\left(u^{\prime}, c\right)$ (by definition of $\left.\Delta_{\mathcal{J}}\left(u, u^{\prime}\right)\right)$, therefore $\neg\left(u I_{A t t} u^{\prime}\right)$. Moreover, if $a \in A t t \backslash C$ then $F(u, a)=F\left(u^{\prime}, a\right)$, thus $u I_{A t t \backslash C} u^{\prime}$. Then we obtain $\pi_{A t t \backslash C}(\mathcal{J}) \neq \pi_{A t t}(\mathcal{J})$, and this proves $(i)$ of Definition 4.1. Let now $D \varsubsetneqq C$ and let us assume by contradiction that $\pi_{A t t \backslash D}(\mathcal{J}) \neq \pi_{A t t}(\mathcal{J})$. So that there exist (at least) two distinct elements $v, w \in U$ such that $v I_{A t t \backslash D} w$ and $\neg\left(v I_{A t t} w\right)$. Since $v I_{A t t \backslash D} w$ we have $F(v, a)=F(w, a)$ for all $a \in A t t \backslash D$, therefore $A t t \backslash D \subseteq A t t \backslash \Delta_{\mathcal{J}}(v, w)$, that is equivalent to $\Delta_{\mathcal{J}}(v, w) \subseteq D$. Let us note that $\Delta_{\mathcal{J}}(v, w) \neq \emptyset$ because $\neg\left(v I_{A t t} w\right)$. We obtain then $\emptyset \neq \Delta_{\mathcal{J}}(v, w) \subseteq D \varsubsetneqq C$, and this is in contrast with the hypothesis that $C$ is an element of $\operatorname{DISC}(\mathcal{J})$ which is minimal with respect to the inclusion. Hence it must be $\pi_{A t t \backslash D}(\mathcal{J})=\pi_{A t t}(\mathcal{J})$ for any $D \varsubsetneqq C$, that is exactly (ii) of Definition 4.1.

Let us note that the equation (22) and the proposition 4.8 are now a direct consequence of the previous theorem.

Corollary 4.12. $\operatorname{CORE}(\mathcal{J})=\{a \in A t t:\{a\} \in D I S C(\mathcal{J})\}$.

Proof. As a consequence of Proposition 4.2 we can identify $C O R E(\mathcal{J})$ with $E S S_{1}(\mathcal{J})$. Moreover, by Theorem 4.11 we know that the elements of $E S S_{1}(\mathcal{J})$ are the minimal subsets of $D I S C(\mathcal{J})$ having one only element, which are exactly all the singletons of $\operatorname{DISC}(\mathcal{J})$.

Example 4.13. Let us consider the following information table J: 


\begin{tabular}{|l|l|l|l|l|}
\hline & $a_{1}$ & $a_{2}$ & $a_{3}$ & $a_{4}$ \\
\hline$u_{1}$ & 0 & 1 & 2 & 0 \\
\hline$u_{2}$ & 1 & 2 & 0 & 2 \\
\hline$u_{3}$ & 1 & 0 & 1 & 0 \\
\hline$u_{4}$ & 2 & 1 & 0 & 1 \\
\hline$u_{5}$ & 1 & 1 & 0 & 2 \\
\hline
\end{tabular}

The discernibility matrix $\Delta[\mathcal{J}]$ is then the following:

\begin{tabular}{|l|l|l|l|l|l|}
\hline & $u_{1}$ & $u_{2}$ & $u_{3}$ & $u_{4}$ & $u_{5}$ \\
\hline$u_{1}$ & $\emptyset$ & $a_{1}, a_{2}, a_{3}, a_{4}$ & $a_{1}, a_{2}, a_{3}$ & $a_{1}, a_{3}, a_{4}$ & $a_{1}, a_{3}, a_{4}$ \\
\hline$u_{2}$ & $*$ & $\emptyset$ & $a_{2}, a_{3}, a_{4}$ & $a_{1}, a_{2}, a_{4}$ & $a_{2}$ \\
\hline$u_{3}$ & $*$ & $*$ & $\emptyset$ & $a_{1}, a_{2}, a_{3}, a_{4}$ & $a_{2}, a_{3}, a_{4}$ \\
\hline$u_{4}$ & $*$ & $*$ & $*$ & $\emptyset$ & $a_{1}, a_{4}$ \\
\hline$u_{5}$ & $*$ & $*$ & $*$ & $*$ & $\emptyset$ \\
\hline
\end{tabular}

Therefore the distinct elements are

$D I S C(\mathcal{J})=\left\{\left\{a_{1}, a_{2}, a_{3}, a_{4}\right\} ;\left\{a_{1}, a_{2}, a_{3}\right\} ;\left\{a_{1}, a_{2}, a_{4}\right\} ;\left\{a_{1}, a_{3}, a_{4}\right\} ;\left\{a_{2}, a_{3}, a_{4}\right\} ;\left\{a_{1}, a_{4}\right\} ;\left\{a_{2}\right\}\right\}$

and the Hasse diagram of the discernibility poset $\mathbb{D}(\mathcal{J})$ is the following:

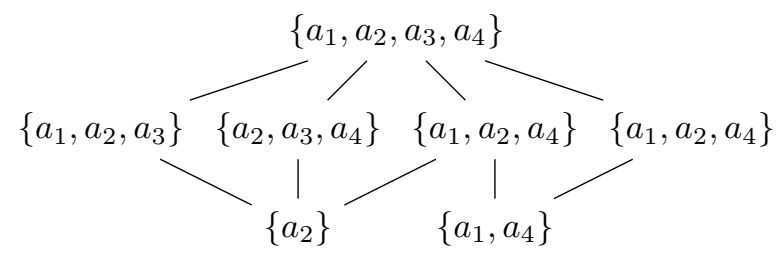

Finally we have that

$$
\operatorname{ESS}(\mathcal{J})=\left\{\left\{a_{2}\right\},\left\{a_{1}, a_{4}\right\}\right\}
$$

and

$$
R E D(\mathcal{J})=\left\{\left\{a_{1}, a_{2}\right\},\left\{a_{2}, a_{4}\right\}\right\}
$$

therefore

$$
\operatorname{CORE}(\mathcal{J})=\left\{a_{2}\right\}
$$

\subsection{Reducts, Core and Hypergraphs}

Firstly, we recall the basic notion of hypergraphs (see [1]).

Definition 4.14. $A$ hypergraph is a pair $H=(X, \mathcal{F})$, where $X=\left\{x_{1}, \ldots, x_{n}\right\}$ is an arbitrary finite set, called vertex set of $H$, and $\mathcal{F}=\left\{Y_{1}, \ldots, Y_{m}\right\}$ is a non-empty family of subsets $Y_{1}, \ldots, Y_{m}$ of $X$. The elements $x_{1}, \ldots, x_{n}$ are called vertices of $H$ and the subsets $Y_{1}, \ldots, Y_{m}$ of $X$ are called hyperedges of $H$. A hypergraph on $X$ is a hypergraph having $X$ as vertex set.

In the next definition we interpret a hypergraph in terms of information tables. 
Definition 4.15. Let $H$ be a hypergraph on a set $X=\left\{x_{1}, \ldots, x_{n}\right\}$, with hyperedges $Y_{1}, \ldots, Y_{m}$. We associate to $H$ an information table $\Gamma(H)$ where the attribute set of $\Gamma(H)$ is $X$ and the universe set of $\Gamma(H)$ is $\left\{Y_{1}, \ldots, Y_{m}\right\}$. The value set is $\{0,1\}$ and the information map $F_{H}$ is defined naturally as

$$
F_{H}\left(Y_{i}, x_{j}\right):= \begin{cases}1 & \text { if } x_{j} \in Y_{i} \\ 0 & \text { otherwise }\end{cases}
$$

Several hypergraphs can be defined taking into account the discernibility matrix, the extended core and the reduct family. In the next definition we explicitly introduce the hypergraphs which we will use in this paper.

Definition 4.16. Given an information table J, we define

- the essential hypergraph of $\mathcal{J}$, the hypergraph $\mathfrak{E}(\mathcal{J}):=(A t t, E S S(\mathcal{J}))$;

- the reduct hypergraph of $\mathcal{J}$, the hypergraph $\mathfrak{R}(\mathcal{J}):=($ Att, $R E D(\mathcal{J}))$;

- the discernibility hypergraph of $\mathcal{J}$, the hypergraph $\mathfrak{D}(\mathcal{J}):=(\operatorname{Att}, \operatorname{DISC}(\mathcal{J}))$;

- $k$-th discernibility hypergraph of $\mathcal{J}$, the hypergraph $\mathfrak{D}_{k}(\mathcal{J}):=\left(\operatorname{Att}, D I S C_{k}(\mathcal{J})\right)$.

Moreover, by analogy with (15) and (16), we also introduce the following terminology:

- if $D I S C_{k}(\mathcal{J}):=\{C \in D I S C(\mathcal{J}):|C|=k\}$, we call discernibility numerical sequence of $\mathcal{J}$ the $n$-tuple $\operatorname{dns}(\mathcal{J}):=\left(\left|D I S C_{1}(\mathcal{J})\right|, \ldots,\left|D I S C_{n}(\mathcal{J})\right|\right)$.

- if $R E D_{k}(\mathcal{J}):=\{C \in R E D(\mathcal{J}):|C|=k\}$, we call reduct numerical sequence of $\mathcal{J}$ the $n$-tuple $r n s(\mathcal{J}):=\left(\left|R E D_{1}(\mathcal{J})\right|, \ldots,\left|R E D_{n}(\mathcal{J})\right|\right)$. We call $k$-reduct of $\mathcal{J}$ a generic element of $R E D_{k}(\mathcal{J})$.

Remark 4.17. Let us remark that, it is possible to find non-isomorphic graphs having the same numerical discernibility matrix and the same discernibility hypergraph. For example, if we consider the graph $F_{4}$ having vertex set $\left\{v_{1}, v_{2}, v_{3}, v_{4}\right\}$ and edge set $\left\{\left\{v_{1}, v_{2}\right\},\left\{v_{3}, v_{4}\right\}\right\}$, it is easy to verify that $\Delta_{n u m}\left(F_{4}\right)=\Delta_{\text {num }}\left(K_{4}\right)$ and $\mathfrak{D}\left(F_{4}\right)=\mathfrak{D}\left(K_{4}\right)$, although $F_{4}$ and $K_{4}$ are not isomorphic graphs. This leads to the interesting open problem of classifying all finite simple graphs by means of their numerical discernibility matrix and their discernibility hypergraph.

We recall now the notion of transversal of a hypergraph (see [1]).

Definition 4.18. Let $H$ be a given hypergraph. A subset $Y \subseteq V(H)$ is said a transversal of $H$ if $Y \cap A \neq \emptyset$ for each non-empty hyperedge $A \in E(H)$. A transversal $A$ of $H$ is said minimal if no proper subset of $A$ is a transversal of $H$. We denote by $\operatorname{Tr}(H)$ the family of all the minimal transversals of $H$. The hypergraph $H^{t r}:=(V(H), \operatorname{Tr}(H))$ is usually called transversal hypergraph of $H$.

In literature the hypergraph transversal problem for $H$ is the problem of generating all the elements of $\operatorname{Tr}(H)$. We adapt the previous terminology in the case of the information tables in the following way: 
Definition 4.19. We call transversal discernibility hypergraph of $\mathcal{J}$ the hypergraph $\mathfrak{D}(\mathcal{J})^{t r}=(A t t, \operatorname{Tr}(\mathfrak{D}(\mathcal{J})))$.

The next result tells us that the reducts of $\mathcal{J}$ are exactly the minimal transversals of the discernibility hypergraph $\mathfrak{D}(\mathcal{J})$.

Theorem 4.20. Let $\mathcal{J}$ be a given information table and $A \subseteq$ Att. Then:

(i) $\pi_{A}(\mathcal{J})=\pi_{A t t}(\mathcal{J})$ if and only if $A$ is a transversal of $\mathfrak{D}(\mathcal{J})$.

(ii) $A$ is a reduct of $\mathcal{J}$ if and only if $A$ is a minimal transversal of $\mathfrak{D}(\mathcal{J})$.

(iii) The hypergraphs $\mathfrak{R}(\mathcal{J})$ and $\mathfrak{D}(\mathcal{J})^{\text {tr }}$ are identical.

Proof. ( $i$ ) Let us note that the equality $\pi_{A}(\mathcal{J})=\pi_{A t t}(\mathcal{J})$ is obviously equivalent to the identity $I_{A}=I_{A t t}$. Therefore, we firstly assume that $I_{A}=I_{A t t}$; we must show that $A$ is a transversal of $\mathfrak{D}(\mathcal{J})$. Let then $D \in D I S C(\mathcal{J})$. By definition of $D I S C(\mathcal{J})$ it results that $D$ is not empty and that there exist two distinct objects $u, u^{\prime} \in U$ such that $D=\{a \in A t t$ : $\left.F(u, a) \neq F\left(u^{\prime}, a\right)\right\}$. Since $D$ contains at least one element, we deduce that $\neg\left(u I_{A t t} u^{\prime}\right)$, and this also imply $\neg\left(u I_{A} u^{\prime}\right)$ from the hypothesis $I_{A}=I_{A t t}$. Hence, by definition of $I_{A}$, we find an element $a^{\prime} \in A$ such that $F\left(u, a^{\prime}\right) \neq F\left(u^{\prime}, a^{\prime}\right)$, i.e. $a^{\prime} \in A \cap D$. This shows that $A \cap D \neq \emptyset$, therefore $A$ is a transversal of $\mathfrak{D}(\mathcal{J})$. We suppose now that $A$ is a transversal of $\mathfrak{D}(\mathcal{J})$ and let $u, u^{\prime}$ be two any distinct objects in $U$. If $u I_{A t t} u^{\prime}$ it is obvious that we also have $u I_{A} u^{\prime}$. We can assume therefore that $\neg\left(u I_{A t t} u^{\prime}\right)$. By definition of $I_{A t t}$ and by (17) it follows then that the attribute subset $D:=\Delta_{\mathcal{J}}\left(u, u^{\prime}\right)$ is not empty, so that $D \in D I S C(\mathcal{J})$. Since $A$ is a transversal of $\mathfrak{D}(\mathcal{J})$ we have that $A \cap D \neq \emptyset$. Let $a^{\prime} \in A \cap D$. Then we obtain an attribute $a^{\prime} \in A$ such that $F\left(u, a^{\prime}\right) \neq F\left(u^{\prime}, a^{\prime}\right)$, and this implies that $\neg\left(u I_{A} u^{\prime}\right)$. Hence $I_{A}=I_{A t t}$.

(ii) Let $A$ be a reduct of $\mathcal{J}$. By definition of reduct we have then $\pi_{A}(\mathcal{J})=\pi_{A t t}(\mathcal{J})$, and by $(i)$ this implies that $A$ is a is a transversal of $\mathfrak{D}(\mathcal{J})$. Now, if $c \in A$, again by definition of reduct we have that $\pi_{A \backslash\{c\}}(\mathcal{J}) \neq \pi_{A t t}(\mathcal{J})$, therefore by $(i)$ it follows that $A \backslash\{c\}$ is not a transversal of $\mathfrak{D}(\mathcal{J})$. Hence $A$ is a minimal transversal of $\mathfrak{D}(\mathcal{J})$. On the other hand, let $A$ be a minimal transversal of $\mathfrak{D}(\mathcal{J})$, then by $(i)$ it follows that $\pi_{A}(\mathcal{J})=\pi_{A t t}(\mathcal{J})$. Now, if $c \in A$, the subset $A \backslash\{c\}$ is not a transversal of $\mathfrak{D}(\mathcal{J})$ by virtue of the minimality of $A$, therefore, again by $(i)$ we obtain $\pi_{A \backslash\{c\}}(\mathcal{J}) \neq \pi_{A t t}(\mathcal{J})$. Hence $A$ is a reduct of $\mathcal{J}$.

(iii) It follows at once by $\left(\right.$ ii) and by definition of the hypergraphs $\mathfrak{R}(\mathcal{J})$ and $\mathfrak{D}(\mathcal{J})^{t r}$.

Let us remark that the minimal transversal problem is widely studied and it has several applications in artificial intelligence $[15,16]$. Therefore, the above result has a double importance from the application standpoint: it connects the reduct problem to other well-known problems and it gives the possibility of using algorithms developed for hypergraphs to find reducts. In particular, in a forthcoming paper, we will discuss how particular cases of the minimal transversal problem apply to reducts, proving the complexity of the reduct problem to be incremental polynomial time [12].

\section{Granular Computing on Three Basic Graph Families}

In this section we apply the theoretical results of Section 4 on some basic graph families. The first two cases that we study are the complete graph $K_{n}$ on $n$ vertices and the complete bipartite graph $K_{p, q}$. Next we examine the case of the undirected path $P_{n}$ on $n$ vertices. 
Proposition 5.1. Let $G=K_{n}$ be the complete graph with vertex set $V(G)=\left\{v_{1}, \ldots, v_{n}\right\}$. Then:

(i) the discernibility matrix of $G$ is $\Delta_{G}\left(v_{i}, v_{j}\right)=\left\{v_{i}, v_{j}\right\}$ for all $i \neq j$;

(ii) the discernibility hypergraph of $G$ is $\mathfrak{D}(G)=\left(\hat{n},\left(\begin{array}{c}\hat{n} \\ 2\end{array}\right)\right)$, therefore dns $(G)=\left(0,\left(\begin{array}{c}n \\ 2\end{array}\right), 0, \ldots, 0\right)$;

(iii) the reduct hypergraph of $G$ is $\mathfrak{R}(G)=\left(\hat{n},\left(\begin{array}{c}\hat{n} \\ n-1\end{array}\right)\right)$, therefore $r n s(G)=(0,0, \ldots, 0, n, 0)$;

(iv) the essential hypergraph of $G$ is $\mathfrak{E}(G)=\left(\hat{n},\left(\begin{array}{c}\hat{n} \\ 2\end{array}\right)\right)$, therefore ens $(G)=\left(0,\left(\begin{array}{l}n \\ 2\end{array}\right), 0, \ldots, 0\right)$ and $\operatorname{Edim}(G)=2$;

$(v)$ the numerical discernibility matrix of $G$ is $\Delta_{\text {num }}[G]=2 \operatorname{Adj}(G)$.

Proof. We identify the set $V(G)=\left\{v_{1}, \ldots, v_{n}\right\}$ with the $n$-element set $\hat{n}$.

(i) By definition of $K_{n}$, for all $v_{i} \in \hat{n}$ we have that $N_{G}\left(v_{i}\right)=\hat{n} \backslash\left\{v_{i}\right\}$. Therefore, for all distinct $v_{i}, v_{j} \in V(G)$ it results by (18) that $\Delta_{G}\left(v_{i}, v_{j}\right)=N_{G}\left(v_{i}\right) \triangle N_{G}\left(v_{j}\right)=\left\{v_{i}, v_{j}\right\}$.

(ii) It follows immediately by $(i)$ and by definition of the discernibility hypergraph $\mathfrak{D}(G)$. (iii) It follows by (ii) and by Theorem 4.20 , because it is immediate to note that the transversal hypergraph of $\left(\hat{n},\left(\begin{array}{c}\hat{n} \\ 2\end{array}\right)\right)$ is exactly $\left(\hat{n},\left(\begin{array}{c}\hat{n} \\ 2\end{array}\right)\right)$.

(iv) It follows by (ii) and by Theorem 4.11, because the hypergraph of all the minimal elements of the discernibility poset $\left(\left(\begin{array}{c}\hat{n} \\ 2\end{array}\right), \subseteq\right)$ is again $\left(\hat{n},\left(\begin{array}{c}\hat{n} \\ 2\end{array}\right)\right)$.

(v) From $(i)$ we have $\delta i j=2$ if $i \neq j$ and $\delta i i=0$. On the other hand, by definition of $K_{n}$ and by definition of adjacency matrix, we also have $\operatorname{Adj}\left(K_{n}\right)=\left(a_{i j}\right)$, where $a_{i j}=1$ if $i \neq j$ and $a_{i i}=0$. Hence $(v)$ follows.

Also for the complete bipartite graph $K_{p, q}$ we can easily obtain the following results.

Proposition 5.2. Let $G=K_{p, q}=\left(B_{1} \mid B_{2}\right)$, where $B_{1}=\left\{x_{1}, \ldots, x_{p}\right\}$ and $B_{2}=$ $\left\{y_{1}, \ldots, y_{q}\right\}$. Let $n=p+q$. Then:

(i) the discernibility matrix of $G$ is $\Delta_{G}\left(x_{i}, x_{i^{\prime}}\right)=\Delta_{G}\left(y_{j}, y_{j^{\prime}}\right)=\emptyset$ and $\Delta_{G}\left(x_{i}, y_{j}\right)=$ $V(G)$ for all $i, i^{\prime} \in\{1, \ldots, p\}, j, j^{\prime} \in\{1, \ldots, q\}$;

(ii) the discernibility hypergraph of $G$ is $\mathfrak{D}(G)=(\hat{n},\{\hat{n}\})$, therefore $\operatorname{dns}(G)=(0,0, \ldots, 0,1)$;

(iii) the reduct hypergraph of $G$ is $\mathfrak{R}(G)=\left(\hat{n},\left(\begin{array}{c}\hat{n} \\ 1\end{array}\right)\right)$, therefore $\operatorname{rns}(G)=(n, 0, \ldots, 0,0)$;

(iv) the essential hypergraph of $G$ is $\mathfrak{E}(G)=(\hat{n},\{\hat{n}\})$, therefore ens $(G)=(0,0, \ldots, 0,1)$ and $\operatorname{Edim}(G)=n$;

(v) the numerical discernibility matrix of $G$ is $\Delta_{\text {num }}[G]=(p+q) \operatorname{Adj}\left(K_{p, q}\right)$.

Proof. As in the previous proposition we identify $V(G)=\left\{x_{1}, \ldots, x_{p}, y_{1}, \ldots, y_{q}\right\}$ with the $n$-element set $\hat{n}$.

(i) When $G=K_{p, q}$ we have $N_{G}\left(x_{i}\right)=B_{2}$ for $i=1, \ldots, p$ and $N_{G}\left(y_{j}\right)=B_{1}$ for $j=$ $1, \ldots, q$. Hence $N_{G}\left(x_{i}\right) \triangle N_{G}\left(x_{i^{\prime}}\right)=\emptyset$ for all $i, i^{\prime} \in\{1, \ldots, p\}$ and $N_{G}\left(y_{j}\right) \triangle N_{G}\left(y_{j^{\prime}}\right)=\emptyset$ for all $j, j^{\prime} \in\{1, \ldots, q\}$. On the other hand, it also results that $N_{G}\left(x_{i}\right) \triangle N_{G}\left(y_{j}\right)=$ $B_{1} \cup B_{2}=V(G)$ for all $i=1, \ldots, p$ and $j=1, \ldots, q$. The thesis follows then by (18).

(ii) It follows immediately by $(i)$ and by definition of the discernibility hypergraph $\mathfrak{D}(G)$. (iii) It follows by (ii) and by Theorem 4.20 , because it is immediate to note that the transversal hypergraph of $(\hat{n},\{\hat{n}\})$ is exactly $\left(\hat{n},\left(\begin{array}{l}\hat{n} \\ 1\end{array}\right)\right)$.

(iv) It follows by (ii) and by Theorem 4.11, because the discernibility poset of $G$ is the singleton $(\{\hat{n}\}, \subseteq)$.

$(v)$ The result follows at once by $(i)$, due to the block form of the adjacency matrix of $K_{p q}$ and by definition of numerical discernibility matrix. 
As a simple corollary of the above propositions we get that both the complete and bipartite complete graphs have empty core.

Corollary 5.3. $\operatorname{CORE}\left(K_{n}\right)=\operatorname{CORE}\left(K_{p, q}\right)=\emptyset$.

Proof. For any simple graph $G$, the core of $G$ can be identified with $E S S_{1}(G)$, moreover $E S S_{1}\left(K_{n}\right)=E S S_{1}\left(K_{p, q}\right)=\emptyset$ by the previous two propositions.

Moreover, we can easily provide a large class of graphs having all empty core. To this aim we recall the following definition.

Definition 5.4. Let $G=(V(G), E(G))$ be a simple undirected graph. $G$ is said vertextransitive if $\operatorname{Aut}(G)$ acts transitively on the set of vertices $V(G)$, i.e. for all $v, v^{\prime} \in V(G)$ there exists $\phi \in \operatorname{Aut}(G)$ such that $\phi(v)=v^{\prime}$.

We have then the following general result.

Proposition 5.5. Let $G$ be a simple undirected vertex-transitive graph. Then $C O R E(G)=$ $\emptyset$.

Proof. Since $\operatorname{Aut}(G)$ acts transitively on $G$, we have that $\left|N_{G}(x)\right|=\left|N_{G}(y)\right|$ for all $x, y \in V(G)$. Thus $\left|\Delta_{G}(x, y)\right|=\left|N_{G}(x) \triangle N_{G}(y)\right|$ is even, for each choice of $x, y$ in $V(G)$. The thesis follows then by Proposition 4.8.

The results obtained so far seem to suggest that graphs with a strong regularity structure have always core number zero. However, we show now a case that contradicts this apparent impression.

Definition 5.6. If $n$ is a positive integer we denote by $P_{n}$ the graph having $n$ vertices $v_{1}, \ldots, v_{n}$ and such that $E\left(P_{n}\right)=\left\{\left\{v_{i}, v_{i+1}\right\}: i=1, \ldots, n-1\right\}$. In literature $P_{n}$ is sometimes called path on $n$ vertices.

Example 5.7. It is immediate to determine all the partitions $\pi_{A}\left(P_{n}\right)$ and to verify that $\operatorname{CORE}\left(P_{n}\right)=\emptyset$ when $1 \leq n \leq 3$. We consider then the path $P_{4}$. If one examine the information table of $P_{4}$ it is easy to verify that $\pi_{\{1,2,3,4\}}=1|2| 3\left|4, \pi_{\{2,3,4\}}=1\right| 3 \mid 24$, $\pi_{\{1,3,4\}}=\pi_{\{1,2,4\}}=1|2| 3 \mid 4$ and $\pi_{\{1,2,3\}}=13|2| 4$. Therefore, the vertices 1 and 4 are indispensable and 2, 3 are not indispensable. Hence $\operatorname{CORE}\left(P_{4}\right)=\{1,4\}$. Analogously, it is easy to verify that $\operatorname{CORE}\left(P_{5}\right)=\{2,4\}, \operatorname{CORE}\left(P_{6}\right)=\{3,4\}, \operatorname{CORE}\left(P_{7}\right)=\{4\}$ and $\operatorname{CORE}\left(P_{8}\right)=\{4,5\}$.

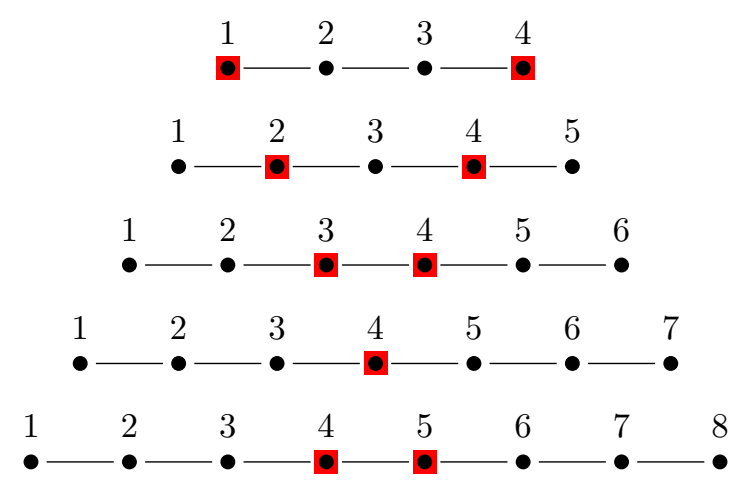

Figure 5: Core of the $n$-Path Graph with $n=4,5,6,7,8$. 
The particular cases discussed in the previous example provide a sequence of cores having a counterintuitive type of symmetry in the core sequences of the paths, therefore in the sequel of this section we examine the general case of the path $P_{n}$, for all $n \geq 1$.

Proposition 5.8. Let $n \geq 4$ and $G=P_{n}$. Then:

$$
\Delta_{G}\left(v_{1}, v_{j}\right)= \begin{cases}\left\{v_{1}, v_{2}, v_{3}\right\} & \text { if } j=2 \\ \left\{v_{4}\right\} & \text { if } j=3 \\ \left\{v_{2}, v_{j-1}, v_{j+1}\right\} & \text { if } 4 \leq j \leq n-1 \\ \left\{v_{2}, v_{n-1}\right\} & \text { if } j=n\end{cases}
$$

and

$$
\Delta_{G}\left(v_{1}, v_{j}\right)= \begin{cases}\left\{v_{i-1}, v_{i}, v_{i+1}, v_{i+2}\right\} & \text { if } j=i+1 \\ \left\{v_{i-1}, v_{i+3}\right\} & \text { if } j=i+2 \\ \left\{v_{i-1}, v_{i+1}, v_{j-1}, v_{j+1}\right\} & \text { if } i+2<j \leq n-1 \\ \left\{v_{i-1}, v_{i+1}, v_{n-1}\right\} & \text { if } j=n, i \neq n-2 \\ \left\{v_{n-3}\right\} & \text { if } j=n, i=n-2\end{cases}
$$

when $2 \leq i<j \leq n$.

Proof. It is a direct consequence of Equation (18), since

$$
N_{G}\left(v_{i}\right)= \begin{cases}\left\{v_{2}\right\} & \text { if } i=1 \\ \left\{v_{4}\right\} & \text { if } 2 \leq i \leq n-1 \\ \left\{v_{n-1}\right\} & \text { if } i=n\end{cases}
$$

In order to give a compact description of the numerical discernibility matrix of $P_{n}$ in the general case, we introduce some new type of matrices.

Definition 5.9. Let $n$ be a positive integer. The matrix $Q_{n}$ is the matrix $Q_{n}=\left(q_{i j}\right)$, where:

$$
q_{i j}:= \begin{cases}1 & \text { if either } i=1 \text { or } j=1 \\ 0 & \text { otherwise. }\end{cases}
$$

Let now $k$ be a positive integer such that $0 \leq k \leq n-1$. The matrix $\operatorname{Diag}(n, k)$ is the matrix $\operatorname{Diag}(n, k)=\left(x_{i j}^{(n, k)}\right)$, where:

$$
x_{i j}^{(n, k)}:= \begin{cases}1 & \text { if }|i-j|=k \\ 0 & \text { otherwise }\end{cases}
$$

We obtain then the following result.

Proposition 5.10. Let $n \geq 4$. Then:

(i) $\Delta_{n u m}\left[P_{n}\right]=4\left(J_{n}-I_{n}\right)-Q_{n}-2 \operatorname{Diag}(n, 2)-\operatorname{Diag}(n, n-1)$.

(ii) $\operatorname{CORE}\left(P_{n}\right)=\left\{v_{4}, v_{n-3}\right\}$.

(iii) $\operatorname{Cnum}\left(P_{n}\right)=2$ for all $n \geq 4$. 
Proof. (i) By (26) and (27) we obtain $\Delta_{\text {num }}\left[P_{n}\right]=\left(\delta_{i j}\right)$, where

$$
\delta_{1 j}= \begin{cases}3 & \text { if } j=2 \text { or } 4 \leq j \leq n-1 \\ 2 & \text { if } j=n \\ 1 & \text { if } j=3\end{cases}
$$

and

$$
\delta_{i j}= \begin{cases}4 & \text { if } j=i+1 \text { or } i+2<j \leq n-1 \\ 3 & \text { if } j=n, i \neq n-2 \\ 2 & \text { if } j=i+2 \\ 1 & \text { if } j=n, i=n-2\end{cases}
$$

when $2 \leq i<j \leq n$. It is easy to check that the previous values of $\delta_{i j}$ are equal to the correspondent entries of the matrix $4\left(J_{n}-I_{n}\right)-Q_{n}-2 \operatorname{Diag}(n, 2)-\operatorname{Diag}(n, n-1)$. The parts (ii) and (iii) follow directly by the previous part (i) and by (24).

Corollary 5.11. If $n \geq 1$ then $\operatorname{ESS}_{1}\left(P_{n}\right)=\left\{\left\{v_{4}\right\},\left\{v_{n-3}\right\}\right\}$. Hence $\operatorname{Edim}\left(P_{n}\right)=1$.

Proof. The result follows directly by (ii) of Proposition 5.10 by virtue of Proposition 4.2 .

Example 5.12. Below we represent the discernibility matrix of $P_{8}$ :

\begin{tabular}{|l|l|l|l|l|l|l|l|l|}
\hline & 1 & 2 & 3 & 4 & 5 & 6 & 7 & 8 \\
\hline 1 & $\emptyset$ & $1,2,3$ & 4 & $2,3,5$ & $2,4,6$ & $2,5,7$ & $2,6,8$ & 2,7 \\
\hline 2 & $*$ & $\emptyset$ & $1,2,3,4$ & 1,5 & $1,3,4,6$ & $1,3,5,7$ & $1,3,6,8$ & $1,3,7$ \\
\hline 3 & $*$ & $*$ & $\emptyset$ & $2,3,4,5$ & 2,6 & $2,4,5,7$ & $2,4,6,8$ & $2,4,7$ \\
\hline 4 & $*$ & $*$ & $*$ & $\emptyset$ & $3,5,4,6$ & 3,7 & $3,5,6,8$ & $3,5,7$ \\
\hline 5 & $*$ & $*$ & $*$ & $*$ & $\emptyset$ & $4,5,6,7$ & 4,8 & $4,6,7$ \\
\hline 6 & $*$ & $*$ & $*$ & $*$ & $*$ & $\emptyset$ & $5,6,7,8$ & 5 \\
\hline 7 & $*$ & $*$ & $*$ & $*$ & $*$ & $*$ & $\emptyset$ & $6,7,8$ \\
\hline 8 & $*$ & $*$ & $*$ & $*$ & $*$ & $*$ & $*$ & $\emptyset$ \\
\hline
\end{tabular}

Hence the numerical discernibility matrix of $P_{8}$ is the following 8-order symmetric matrix:

$$
\left[\begin{array}{llllllll}
0 & 3 & 1 & 3 & 3 & 3 & 3 & 2 \\
3 & 0 & 4 & 2 & 4 & 4 & 4 & 3 \\
1 & 4 & 0 & 4 & 2 & 4 & 4 & 3 \\
3 & 2 & 4 & 0 & 4 & 2 & 4 & 3 \\
3 & 4 & 2 & 4 & 0 & 4 & 2 & 3 \\
3 & 4 & 4 & 2 & 4 & 0 & 4 & 1 \\
3 & 4 & 4 & 4 & 2 & 4 & 0 & 3 \\
2 & 3 & 3 & 3 & 3 & 1 & 3 & 0
\end{array}\right]
$$

\section{Granular Computing on the Petersen Graph}

In this section, we apply all the general notions developed in the previous sections on a specific case of study: the Petersen graph, abbreviated Pet. It is well known that the Petersen graph is both vertex-transitive and edge-transitive. 
Remark 6.1. Let us note that in the Petersen graph do not occur cycles of lenght 3 and 4.

Proposition 6.2. Let $G=$ Pet. Then

$$
\delta_{i j}=:\left|\Delta_{G}\left(v_{i}, v_{j}\right)\right|= \begin{cases}6 & \text { if } v_{i} \sim v_{j}, \\ 4 & \text { otherwise. }\end{cases}
$$

Proof. It results that $N_{G}\left(v_{1}\right)=\left\{v_{2}, v_{5}, v_{6}\right\}, N_{G}\left(v_{2}\right)=\left\{v_{1}, v_{3}, v_{7}\right\}, N_{G}\left(v_{3}\right)=\left\{v_{2}, v_{4}, v_{8}\right\}$, $N_{G}\left(v_{4}\right)=\left\{v_{3}, v_{5}, v_{9}\right\}, N_{G}\left(v_{5}\right)=\left\{v_{1}, v_{4}, v_{10}\right\}, N_{G}\left(v_{6}\right)=\left\{v_{1}, v_{8}, v_{9}\right\}, N_{G}\left(v_{7}\right)=\left\{v_{2}, v_{9}, v_{10}\right\}$, $N_{G}\left(v_{8}\right)=\left\{v_{3}, v_{6}, v_{10}\right\}, N_{G}\left(v_{9}\right)=\left\{v_{4}, v_{6}, v_{7}\right\}, N_{G}\left(v_{10}\right)=\left\{v_{5}, v_{7}, v_{8}\right\}$. We can see therefore that $N_{G}\left(v_{1}\right) \cap N_{G}\left(v_{i}\right)=\emptyset$ for $i=2,5,6$, that are exactly the vertices adjacent to $v_{1}$, and $N_{G}\left(v_{1}\right) \cap N_{G}\left(v_{i}\right)$ is a singleton for the vertices $v_{i}$ which are not adjacent to $v_{1}$. A similar situation occurs if we examine the vertex $v_{6}$ in place of $v_{1}$. By virtue of the symmetric form of the Petersen graph, the vertices $v_{2}, v_{3}, v_{4}, v_{5}$ will have the same behavior of $v_{1}$, whereas the vertices $v_{7}, v_{8}, v_{9}, v_{10}$ will have the same behavior of $v_{6}$. Hence, for all $v_{i}, v_{j} \in V(G)$ we deduce that either $v_{i} \sim v_{j}$ and the vertex subset $\Delta_{G}\left(v_{i}, v_{j}\right)$ contains six vertices or otherwise it contains four vertices. Therefore the thesis follows.

Corollary 6.3. (i) Cnum $($ Pet $)=0$.

(ii) $\Delta_{\text {num }}[$ Pet $]=4\left(J_{10}-I_{10}\right)+2 \operatorname{Adj}($ Pet $)$.

Proof. (i) The thesis follows by (25) and Proposition 6.2.

(ii) It follows by Proposition 6.2 and by the definition of the adjacency matrix.

Proposition 6.4. Let $G=$ Pet and let $v_{i}, v_{j} \in V(G)$ such that $v_{i} \sim v_{j}$. Then:

$$
\Delta_{G}\left(v_{i}, v_{j}\right)=V(G) \backslash \Delta_{G}\left(x_{i}, y_{i}\right)=V(G) \backslash \Delta_{G}\left(x_{j}, y_{j}\right),
$$

where $\left\{x_{i}, y_{i}\right\}=N_{G}\left(v_{i}\right) \backslash\left\{v_{j}\right\}$ and $\left\{x_{j}, y_{j}\right\}=N_{G}\left(v_{j}\right) \backslash\left\{v_{i}\right\}$.

Proof. By Remark 6.1 we have that $x_{i} \nsim y_{i}$ and $x_{j} \nsim y_{j}$.

Let us prove the first equality, the other follows similarly. Thus, by Proposition 6.2 $\left|\Delta_{G}\left(v_{i}, v_{j}\right)\right|=6$ and $\left|\Delta_{G}\left(x_{i}, y_{i}\right)\right|=\left|\Delta_{G}\left(x_{j}, y_{j}\right)\right|=4$. So it is sufficient to prove one of the two inclusions.

We have $N_{G}\left(v_{i}\right)=\left\{v_{j}, x_{i}, y_{i}\right\}$ and $N_{G}\left(v_{j}\right)=\left\{v_{i}, x_{j}, y_{j}\right\}$, so $\Delta_{G}\left(v_{i}, v_{j}\right)=\left\{v_{i}, v_{j}, x_{i}, y_{i}, x_{j}, y_{j}\right\}$.

Let $u \in \Delta_{G}\left(v_{i}, v_{j}\right)$ and let us show that $u \notin \Delta_{G}\left(x_{i}, y_{i}\right)$. If $u=v_{i}$, then $u \in$ $N_{G}\left(x_{i}\right) \cap N_{G}\left(y_{i}\right)$, so $u \notin N_{G}\left(x_{i}\right) \triangle N_{G}\left(y_{i}\right)=\Delta_{G}\left(x_{i}, y_{i}\right)$. If $u=v_{j}$, then, by Remark 6.1, $u \notin N_{G}\left(x_{i}\right)$ and $u \notin N_{G}\left(y_{i}\right)$, so $u \notin \Delta_{G}\left(x_{i}, y_{i}\right)$. Now, by Remark 6.1 we deduce that $\left\{x_{i}, y_{i}, x_{j}, y_{j}\right\} \cap N_{G}\left(x_{i}\right)=\left\{x_{i}, y_{i}, x_{j}, y_{j}\right\} \cap N_{G}\left(y_{i}\right)=\emptyset$ and thus $u \notin \Delta_{G}\left(x_{i}, y_{i}\right)$, therefore the thesis follows.

Corollary 6.5. Let $G=$ Pet and $B \subseteq V(G)$. Then $B \in D I S C_{6}(G)$ if and only if $V(G) \backslash B \in D I S C_{4}(G)$.

Proof. By Proposition 6.2, $B \in D I S C_{6}(G)$ if and only if $B=\Delta_{G}\left(v_{i}, v_{j}\right)$, where $v_{i}, v_{j} \in$ $V(G)$ and $v_{i} \sim v_{j}$. By Proposition 6.4 this is equivalent to say that $V(G) \backslash B \in$ $\mathrm{DISC}_{4}(G)$. 
For its symmetry properties the core of the Petersen graph is empty. The key and a priori not obvious point is the characterization of the reducts of the Petersen graph from a geometric point of view as follows.

Theorem 6.6. Let $G=$ Pet. A subset $A \subseteq V(G)$ is a reduct of $G$ if and only if $|A|=5$ and $G[A]$ is isomorphic to one of the following graphs:
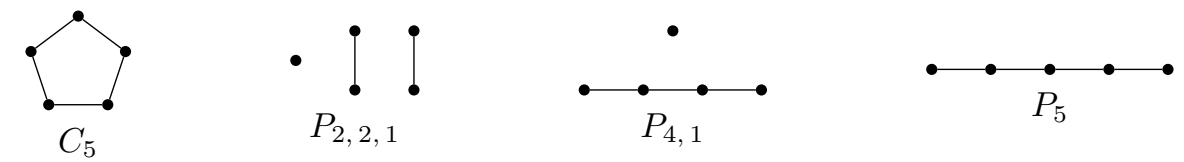

Moreover $B \subseteq V(G)$ is an essential subset of $G$ if and only if $|B|=4$ or $|B|=6$ and $G[B]$ is isomorphic to one of the following graphs:
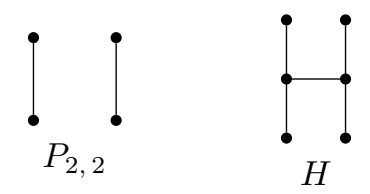

Before proving Theorem 6.6, we will establish several preliminary results:

1. Firstly we will characterize geometrically the hyperedges in $D I S C_{4}(G)$ (Proposition $6.7)$.

2. Then we will find the geometric properties of the subsets of $V(G)$ which are not transversals of the hypergraph $\mathfrak{D}_{4}(G)=\left(V(G), D I S C_{4}(G)\right)$ (Proposition 6.8).

3. Next we will prove that any transversal of the discernibility hypergraph $\mathfrak{D}(G)$ has cardinality greater than or equal to 5 (Proposition 6.9).

4. Finally we will prove the Theorem 6.6.

Proposition 6.7. Let $G=$ Pet and $B \subseteq V(G)$. Then $B \in D I S C_{4}(G)$ if and only if $G[B]$ is isomorphic to the following graph:

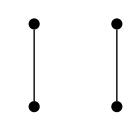

Proof. Let $\mathcal{B}$ be the set of all 4 -subsets $B$ of $V(G)$ such that $G[B]$ is isomorphic to the previous pattern. Let us compute the cardinality $b$ of $\mathcal{B}$. The elements in $\mathcal{B}$ containing the vertices 1,2 are $\{1,2,4,9\}$ and $\{1,2,8,10\}$. Since Pet is edge-transitive, each pair of adjacent vertices in Pet occurs twice in some of the elements in $\mathcal{B}$. So we obtain

$$
b=2|E(G)| \frac{1}{2}=|E(G)|=15 .
$$

Then, by the previous proposition, $b=|\mathcal{B}|=\left|D I S C_{4}(G)\right|$.

Let us prove now that $\mathcal{B} \subseteq D I S C_{4}(G)$. Let $B=\left\{v_{1}, v_{2}, u_{1}, u_{2}\right\} \in \mathcal{B}$ such that $v_{1} \sim v_{2}$ and $u_{1} \sim u_{2}$. Since $v_{1} \nsim u_{1}$ and $v_{2} \nsim u_{2}$, there exist $x_{1}, x_{2} \in V(G)$ such that $v_{1} \sim x_{1}, u_{1} \sim x_{1}, v_{2} \sim x_{2}$ and $u_{2} \sim x_{2}$. By Remark 6.1, $x_{1} \nsim x_{2}$, so there exists a vertex $y \in V(G)$ such that $x_{1} \sim y$ and $x_{2} \sim y$. It follows that $\Delta_{G}\left(x_{1}, x_{2}\right)=N_{G}\left(x_{1}\right) \triangle N_{G}\left(x_{2}\right)=$ $\left\{v_{1}, u_{1}, y\right\} \triangle\left\{v_{2}, u_{2}, y\right\}=B$. In picture: 


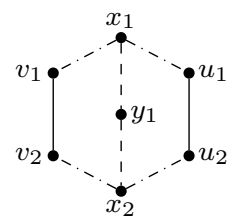

Proposition 6.8. Let $G=$ Pet. A set of vertices $C \subseteq V(G)$ is not a transversal of the hypergraph $\mathfrak{D}_{4}(G)$ if and only if there exists a 6-subset $D$ of $V(G)$ such that $C \subseteq D$ and $G[D]$ is isomorphic to a subgraph of the following graph $H$ :

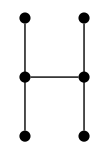

Proof. Let us show first that if $B=\left\{u_{1}, u_{2}, v_{1}, v_{2}\right\} \in \mathcal{B}$ (B defined as in the previous proposition), where $u_{1} \sim u_{2}$ and $v_{1} \sim v_{2}$, then $G[V(G) \backslash B]$ is isomorphic to the graph in the statement.

As before, there exist three vertices $x_{1}, x_{2}, y_{1} \in V(G) \backslash B$ such that $G\left[B \cup\left\{x_{1}, x_{2}, y_{1}\right\}\right]$ is the following graph:

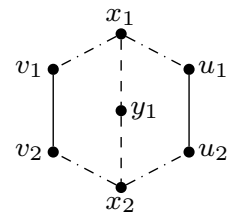

Since $\left|N_{G}(v)\right|=3$ for all $v \in V(G)$, there exist vertices (not necessarely distinct) $w_{1}, w_{2}, z_{1}, z_{2}, y_{2} \in V(G)$ such that $u_{1} \sim w_{1}, u_{2} \sim w_{2}, v_{1} \sim z_{1}, v_{2} \sim z_{2}$, and $y_{1} \nsim y_{2}$. By Remark 6.1, $y_{2} \notin\left\{w_{1}, w_{2}, z_{1}, z_{2}\right\}, w_{1} \neq w_{2}, w_{1} \neq z_{1}, w_{2} \neq z_{2}$ and $z_{1} \neq z_{2}$.

Since $|V(G)|=10$, we obtain $w_{1}=z_{2}$ and $w_{2}=z_{1}$. So the Petersen graph can be represented as in the following figure.

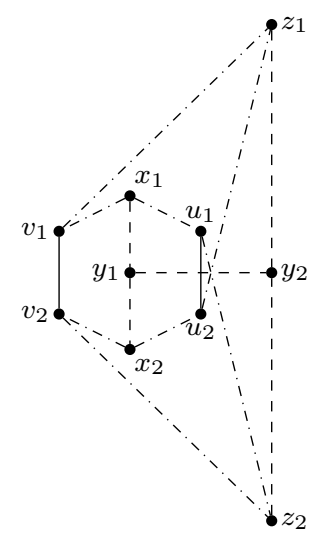


Then $G[V(G) \backslash B]$ is isomorphic to $H$ because $V(G) \backslash B=\left\{x_{1}, x_{2}, y_{1}, y_{2}, z_{1}, z_{2}\right\}$.

Let $C \subseteq V(G)$.

By Proposition 6.7, $C$ is not a transversal of the hypergraph $\left(V(G), D I S C_{4}(G)\right)$ if and only if there exists $B \in \mathcal{B}$ such that $C \cap B=\emptyset$. This shows that if $C$ is not a transversal of the hypergraph $\mathfrak{D}_{4}(G)$ then $C \subseteq D:=V(G) \backslash B$ and $G[D]$ is isomorphic to the graph in the statement.

Let us assume now that $C \subseteq D=\left\{x_{1}, x_{2}, y_{1}, y_{2}, z_{1}, z_{2}\right\} \subseteq V(G)$ such that $G[D]$ is:

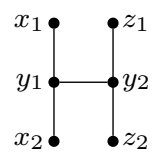

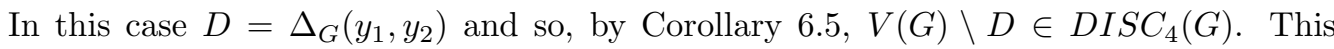
proves the proposition.

Proposition 6.9. Let $G=$ Pet. If $B \subseteq V(G)$ is a transversal of the hypergraph $\mathfrak{D}_{4}(G)$, then either $B \in D I S C_{4}(G)$ or $|B| \geq 5$.

Proof. Let $B \subseteq V(G)$ such that $|B|=4$ and $B \notin D I S C_{4}(G)$. By Remark $6.1, G[B]$ is a graph on 4 vertices with no cycles. Thus, by Proposition 6.7, it is isomorphic to one of the following graphs:
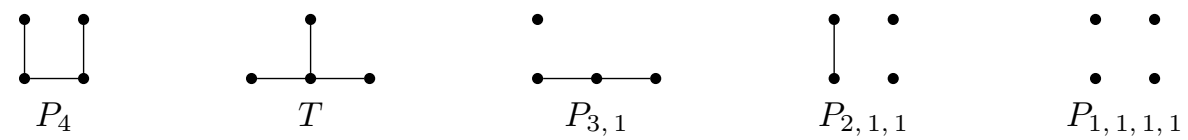

We prove now that, in all the previous cases, there exist two vertices $x, y$ in $V(G) \backslash B$ such that $G[B \cup\{x, y\}]$ is isomorphic to $H$. As a consequence, by Proposition 6.8 we deduce that $B$ is not a trasnversal of $\mathfrak{D}_{4}(G)$.

CASE (I) Let $B=\left\{v_{1}, v_{2}, v_{3}, v_{4}\right\} \subseteq V(G)$ such that $G[B]$ is the following graph:

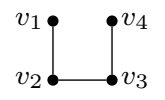

Since $\left|N_{G}(v)\right|=3$, for any vertex $v \in V(G)$, let $x$ and $y$ be the unique vertices in $N_{G}\left(v_{2}\right) \backslash B$ and $N_{G}\left(v_{3}\right) \backslash B$ respectively. Since there are no cycles of lenght 3 in the Petersen graph, both $x$ and $y$ are not adjacent to any other vertex in $B$. Thus $G[B \cup$ $\{x, y\}]$ is

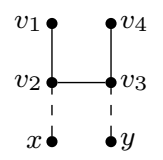

which is clearly isomorphic to $H$.

CASE (II) Let $B=\left\{v_{1}, v_{2}, v_{3}, v_{4}\right\} \in V(G)$ such that $G[B]$ is the following graph: 


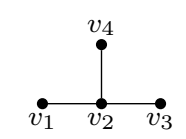

Since $\left|N_{G}(v)\right|=3$, for any vertex $v \in V(G)$, let $x$ and $y$ be the vertices in $N_{G}\left(v_{4}\right) \backslash B$. By Remark 6.1 again, $x$ and $y$ are not adjacent to any other vertex in $B$. Thus $G[B \cup\{x, y\}]$ is

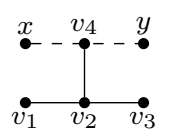

which is isomorphic to $H$.

CASE (III) Let $B=\left\{v_{1}, v_{2}, v_{3}, v_{4}\right\} \in V(G)$ such that $G[B]$ is the following graph:

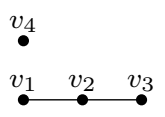

Since $v_{2}$ is not adjacent to $v_{4}$, there exist a vertex $x \in V(G) \backslash B$ such that $x$ is adjacent to both vertices $v_{2}$ and $v_{4}$. Let $y \in N_{G}(x) \backslash B$ the other vertex in $V(G)$ adjacent to $x$. By Remark 6.1 again, $x$ and $y$ are not adjacent to any other vertex in $B$. Thus $G[B \cup\{x, y\}]$ is

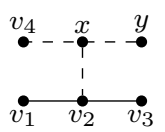

which is isomorphic to $H$.

CASE (IV) Let $B=\left\{v_{1}, v_{2}, v_{3}, v_{4}\right\} \in V(G)$ such that $G[B]$ is the following graph:

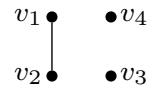

Since $v_{1}$ and $v_{2}$ are not adjacent to both vertices $v_{3}$ and $v_{4}$, there exist vertices $u_{1}, u_{2}, u_{3}, u_{4}$ in $V(G)$ such that $u_{1} \in N_{G}\left(v_{1}\right) \cap N_{G}\left(v_{4}\right), u_{2} \in N_{G}\left(v_{1}\right) \cap N_{G}\left(v_{3}\right), u_{3} \in N_{G}\left(v_{2}\right) \cap N_{G}\left(v_{4}\right)$ and $u_{4} \in N_{G}\left(v_{2}\right) \cap N_{G}\left(v_{3}\right)$. By Remark 6.1, each of the vertices in $\left\{u_{1}, u_{2}\right\}$ is not in $\left\{u_{3}, u_{4}\right\}$. Let us suppose that the vertices $u_{1}, u_{2}, u_{3}, u_{4}$ are all distinct. In figure:

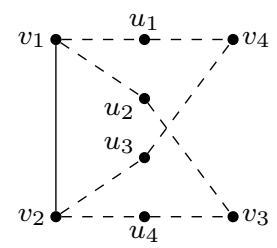

Since $u_{1}$ is not adjacent to $u_{4}$, there exists a vertex $w_{1}$ such that $u_{1} w_{1}, u_{4} w_{1} \in E(G)$. Similarly there exists a vertex $w_{2} \in V(G)$ adjacent to both $u_{2}$ and $u_{3}$. Since each vertex of the Petersen graph has degree 3 , we have $w_{1} \neq w_{2}$. Moreover $w_{1}$ and $w_{2}$ are not in $\left\{v_{1}, v_{2}, v_{3}, v_{4}, u_{1}, u_{2}, u_{3}, u_{4}\right\}$ because in that case, there would exist a cycle with length 
less than 5 in $G$. Thus $V(G)=\left\{v_{1}, v_{2}, v_{3}, v_{4}, u_{1}, u_{2}, u_{3}, u_{4}, w_{1}, w_{2}\right\}$. The extended graph becomes:

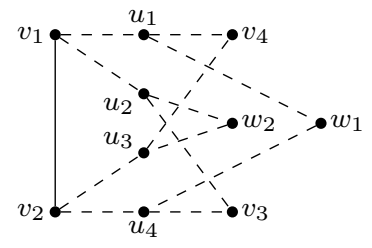

Let us consider the vertex $v_{3}$. Since its degree is equal to 3 , it must exist a vertex in $V(G) \backslash\left\{u, u_{3}\right\}$ that is adjacent to $v_{3}$. But this is impossible still by Remark 6.1. Thus $u_{1}=u_{2}$ or $u_{3}=u_{4}$. If $u_{1}=u_{2}\left(u_{3}=u_{4}\right)$, then, by setting $x:=u_{1}=u_{2}\left(x:=u_{3}=u_{4}\right)$ and $y$ equal to the third vertex in $N_{G}\left(v_{1}\right)\left(N_{G}\left(v_{2}\right)\right)$ different from $x$ and $v_{2}\left(x\right.$ and $\left.v_{1}\right)$, then $G[B \cup\{x, y\}]$ is the following graph:
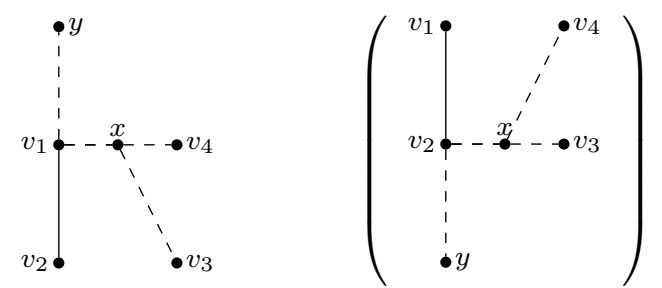

which is isomorphic to $H$.

CASE (V) Let $B=\left\{v_{1}, v_{2}, v_{3}, v_{4}\right\} \in V(G)$ such that $G[B]$ is the following graph:
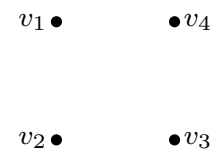

In this case there exist vertices $x_{1}, x_{2}, y_{1}, y_{2}, z_{1}, z_{2} \in V(G)$ such that the corresponding extended graph is the following:

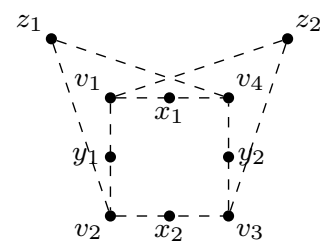

They are all distinct because, if $x_{1}=x_{2}, y_{1}=y_{2}$ or $z_{1}=z_{2}$, then some vertices would have degree 4 , and this does not occur in the Petersen graph. If another equality between two of them holds, then there would exist a vertex $w$ in $\left\{x_{1}, x_{2}, y_{1}, y_{2}, z_{1}, z_{2}\right\}$ such that $N_{G}(w) \subset\left\{v_{1}, v_{2}, v_{3}, v_{4}\right\}$. In this case the six vertices different from $w$ adjacent to one of the three vertices in $N_{G}(w)$ are all different and thus in such a case it would be an edge between two of the vertex in $\left\{v_{1}, v_{2}, v_{3}, v_{4}\right\}$. Then, since $\left|N_{G}(v)\right|=3$ for each $v \in$ $V(G)$, by Remark 6.1 we deduce that the unique possibility is $x_{1} x_{2}, y_{1} y_{2}, z_{1} z_{2} \in E(G)$. Graphically: 


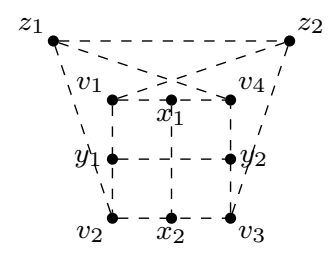

Then the three 6-subsets of $V(G), C_{1}=B \cup\left\{x_{1}, x_{2}\right\}, C_{2}=B \cup\left\{y_{1}, y_{2}\right\}$ and $C_{3}=$ $B \cup\left\{z_{1}, z_{2}\right\}$ contain $B$ and the subgraphs of $G$ induced by $C_{1}, C_{2}$ and $C_{3}$ are isomorphic to $H$.

Corollary 6.10. Let $G=$ Pet and let $A \subseteq V(G)$. A is a transversal of the discernibility hypergraph $\mathfrak{D}(G)$ if and only if $A$ is a transversal of $\mathfrak{D}_{4}(G)$ and $A \notin D I S C_{4}(G)$. In particular each reduct of $G$ has cardinality greater than or equal to 5 .

Proof. Let $A \subseteq V(G)$ be a transversal of $\mathfrak{D}(G)$. Since $D I S C_{4}(G) \subseteq D I S C(G)$, the subset $A$ is a transversal of $\mathfrak{D}_{4}(G)$. Then, if $A \in D I S C_{4}(G)$, by Corollary 6.5 we obtain $V(G) \backslash A \in D I S C(G)$, that is in contrast with the assumption on $A$.

Let now $A \subseteq V(G)$ be a transversal of $\mathfrak{D}_{4}(G)$ and we assume that $A \notin D I S C_{4}(G)$. Then, by Proposition $6.9|A| \geq 5$. Let $D \in D I S C(G)$. By Proposition 6.2 we have $|D|=4$ or $|D|=6$. If $|D|=4$, then $D \in D I S C_{4}(G)$ and thus $D \cap A \neq \emptyset$. If $|D|=6$, since $|A| \geq 5$ and $|V(G)|=10$, we obtain $D \cap A \neq \emptyset$. So $A$ is a transversal of $\mathfrak{D}(G)$.

The last assertion follows directly from the definition of reduct.

We are ready now to prove Theorem 6.6.

Proof of Theorem 6.6. Firstly, let us note that, if $B_{1}$ and $B_{2}$ are different subsets of $V(G)$ such that the corresponding induced subgraphs are isomorphic to $H$ (see 6.8), then $\left|B_{1} \cap B_{2}\right| \leq 4$. To start, we will prove that if $A \subseteq V(G)$ has five vertices and $G[A]$ is not isomorphic to any of the graphs listed in the statement, then $G[A]$ it is isomorphic to one of the following two graphs:

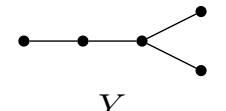

$Y$

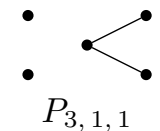

To this aim let us count the number of 5-subsets $A$ of $V(G)$ such that the induced subgraph $G[A]$ is isomorphic to one of the graphs $C_{5}, P_{2,2,1}, P_{4,1}, P_{5}, Y$ or $P_{3,1,1}$. Let $v \in V(G)$ be a fixed vertex.

Case $G[A] \equiv C_{5}$. The 5 -subsets of $V(G)$ containing $v$ must contain exactly two vertices $w_{1}, w_{2} \in N_{G}(v)$. For each choice of such vertices, there exist two edges in $G$ joining a vertex in $N_{G}\left(w_{1}\right) \backslash v$ with a vertex in $N_{G}\left(w_{2}\right) \backslash v$. Thus there exist two subsets of $V(G)$ containing $v, w_{1}, w_{2}$ whose induced subgraph is a cycle of lenght 5 and so six of these subsets contain $v$. It follows that the sought number is equal to

$$
n_{c}=\frac{6|V(G)|}{5}=12 .
$$

Case $G[A] \equiv P_{2,2,1}$. It is straightforward to see that $G\left[V(G) \backslash\left(\{v\} \cup N_{G}(v)\right)\right]$ is a cycle of length 6 . So there exist three subsets of $V(G)$ whose corresponding induced 
graph is isomorphic to that in $(b)$ and where the isolated vertex is $v$. It follows that 305 is the total number of 5 -subsets of $V(G)$ whose induced subgraph is isomorphic to $P_{2,2,1}$.

Case $G[A] \equiv P_{4,1}$. As we proved before, $G\left[V(G) \backslash\left(\{v\} \cup N_{G}(v)\right)\right]$ is a cycle of length 6. Such a cycle contain six different path graphs of length 4 . Thus there are six subsets of $V(G)$ whose corresponding induced graph is isomorphic to that in $(c)$ and where the isolated vertex is $v$ and finally the sought number is 60 .

Case $G[A] \equiv P_{5}$. The number of 5 -subsets of $V(G)$ whose induced subgraph is isomorphic to the graph in $(d)$ and having $v$ as the "central" vertex is equal to 6 . To prove this, let us note that such a subset must contain exactly two vertices $w_{1}, w_{2} \in N_{G}(v)$ and two more vertices $u_{1}, u_{2}$, not each other adjacent, one in $N_{G}\left(w_{1}\right) \backslash\{v\}$ and the other in $N_{G}\left(w_{2}\right) \backslash\{v\}$. It is easy to see that there are three possible choices for $w_{1}, w_{2}$ and then two choices for $u_{1}, u_{2}$. Since $G$ is vertex-transitive, there are six subsets $A$ of $V(G)$ whose induced subgraph is isomorphic to $P_{5}$ having $v$ in the central position. Thus the sought number is equal to 60 .

Case $G[A] \equiv Y$. The number of 5 -subsets of $V(G)$ whose induced subgraph is isomorphic to the graph in $(e)$ and having $v$ as the vertex of degree 3 is equal to 6 . In fact such a subset must contain $v, N_{G}(v)$ and one of the other vertex which is clearly adjacent to one vertex in $N_{G}(v)$. Since $G$ is vertex-transitive, the sought number is equal to $6|V(G)|=60$.

Case $G[A] \equiv P_{3,1,1}$. Let us count the number of 5 -subsets of $V(G)$ containing $v$ whose induced subgraph is isomorphic to the graph in $A$ and having $v$ as the vertex of degree 2. Let $w_{1}, w_{2}$ be the two vertices in $A$ which are adjacent to $v$ and let $u$ the other vertex in $N_{G}(v)$. A must contain the two vertices in $N_{G}(u)$ different from $v$ because the other vertices are adjacent to $w_{1}$ or $w_{2}$ and this contradicts our assumption. So there is one such subset for each choice of $w_{1}, w_{2} \in N_{G}(v)$. Thus there are three subsets $A$ of $V(G)$ whose vertex with degree 2 is $v$ and by symmetry of the Petersen graph there are thirty 5-subsets of $V(G)$ whose induced subgraph is isomorphic to the graph in $P_{3,1,1}$.

The sum of all these numbers is exactly 252 , that is equal to $\left(\begin{array}{c}10 \\ 5\end{array}\right)$. So the assert holds.

If $A$ is a subset of $G$ such that $G[A]$ is isomorphic to a graph among $C_{5}, P_{2,2,1}, P_{4,1}$ and $P_{5}$, then there is no vertex subset $B$ of $G$ such that $A \subseteq B$ and $G[B] \equiv H$. Hence $A$ is a transversal of $\mathfrak{D}_{4}(G)$ and thus, by Proposition 6.8 , it is a transversal of $\mathfrak{D}(G)$.

Let now $A \subseteq V(G)$ be such that $G[A] \equiv C_{5}$ and let $v \in A$ having degree 2 in $G[A]$. Let $w$ the third vertex in $N_{G}(v)$ which is not in $A$. Then $G[A \cup\{u\}]$ is clearly isomorphic to $H$. Thus $A$ is not a transversal of $\mathfrak{D}_{4}(G)$ and so it can not be a reduct of $G$. Similarly, if $G[A]$ is isomorphic to $P_{2,2,1}$ and $v$ is the vertex in $A$ having degree 2 , then $A \backslash\{v\}$ is isomorphic to the empty graph on four vertices and therefore, as in the proof of Proposition 6.9 (CASE $(\mathrm{V})$ ), the vertex $w \in V(G)$ adjacent to the isolated vertices in $A$ is adjacent to $v$ too, hence $G[A \cup\{u\}]$ is isomorphic to $H$. By Proposition $6.8, A$ is not a reduct of $G$.

Now, if $B \subseteq V(G)$ is a transversal of $\mathfrak{D}_{4}(G)$ such that $|B| \geq 6$, we prove that $B$ contains a 5 -subset of $V(G)$ whose induced subgraph is one among $C_{5}, P_{2,2,1}, P_{4,1}$ and $P_{5}$. It is trivially sufficient to prove this fact for the vertex subsets $B$ having six 
elements such that $G[B]$ is not isomorphic to $H$. Let $B=\left\{v_{1}, v_{2}, v_{3}, v_{4}, v_{5}, v_{6}\right\}$ such a subset and let $A=\left\{v_{1}, v_{2}, v_{3}, v_{4}, v_{5}\right\}$. Let us suppose $G[A] \equiv Y$. In figure:

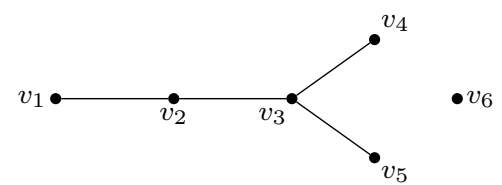

The vertex $v_{6}$ is not adjacent to the vertices $v_{2}, v_{3}$ because, in the first case $G[B]$ would be isomorphic to $H$, in the second case the vertex $v_{3}$ would have degree at least 4 . Thus $G\left[\left\{v_{1}, v_{2}, v_{3}, v_{4}, v_{6}\right\}\right]$ is isomorphic to one of the following graphs:

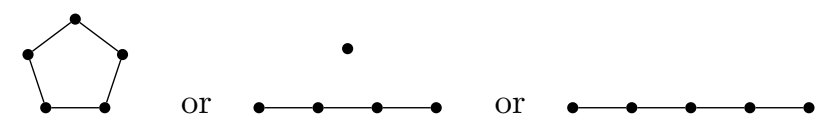

Thus $B$ is not a reduct.

Let $B=\left\{v_{1}, v_{2}, v_{3}, v_{4}, v_{5}, v_{6}\right\}$ and $A=\left\{v_{1}, v_{2}, v_{3}, v_{4}, v_{5}\right\}$ as before such that $G[A] \equiv P_{3,1,1}$. In figure:

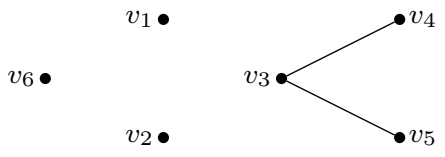

If $v_{6}$ was not adjacent to any of the vertices in $\left\{v_{1}, v_{2}, v_{4}, v_{5}\right\}$, then $G\left[\left\{v_{1}, v_{2}, v_{4}, v_{5}, v_{6}\right\}\right]$ would be the empty graph on 5 vertices, and this is not possible as we proved before. So $v_{6}$ is adjacent to at least one of the vertices in $\left\{v_{1}, v_{2}, v_{4}, v_{5}\right\}$.

If $v_{6}$ is adjacent to $v_{4}$ (or $v_{5}$ ), then $G\left[\left\{v_{1}, v_{3}, v_{4}, v_{5}, v_{6}\right\}\right]$ (or $G\left[\left\{v_{2}, v_{3}, v_{4}, v_{5}, v_{6}\right\}\right]$ ) is isomorphic, as before, to one of the following graphs:
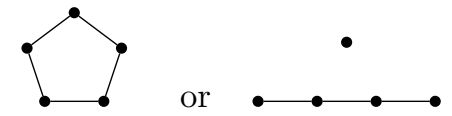

or

Let us suppose that $v_{6}$ is not adjacent to both $v_{4}$ and $v_{5}$. Then, if $v_{6}$ is adjacent to $v_{1}$, the subgraph $G\left[\left\{v_{1}, v_{3}, v_{4}, v_{5}, v_{6}\right\}\right]$ is isomorphic to the following graph:

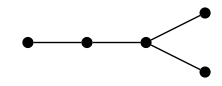

and thus also $G\left[\left\{v_{2}, v_{3}, v_{4}, v_{5}, v_{6}\right\}\right]$ is isomorphic to the same graph. This is impossible because in that case the above subgraph of $G$ would be isomorphic to $H$, contradicting the assumption. Similarly $v_{3}$ can not be adjacent to $v_{2}$. Thus $B$ is not a reduct.

For the second part of the statement of Theorem 6.6, we note that if $A \in D I S C_{4}(G)$ then $G[A]$ is isomorphic to $Y$, by virtue of Proposition 6.7. Moreover, by Propositions 6.4 and 6.8, if $D \in D I S C_{6}(G)$ then $G[D]$ is isomorphic to $P_{3,1,1}$. In fact, if $D \in D I S C_{6}(G)$, by Proposition 6.4 we deduce that $V(G) \backslash D \in D I S C_{4}(G)$. Thus $D$ is not a transversal of the hypergraph $\mathfrak{D}_{4}(G)$ and, by Proposition $6.8, G[D]$ is isomorphic to $P_{3,1,1}$. On the other hand, let $D \in D I S C_{6}(G)$. Since no 4 -subset $A$ of $D$ is such that $G[A] \equiv Y$, then $D$ is minimal with respect to the inclusion relation. Thus any hyperedge of the 
discernibility hypergraph is an essential subset of the Petersen graph. This concludes the proof of Theorem 6.6.

Corollary 6.11. Let $G=$ Pet. Then:

(i) $|\operatorname{RED}(G)|=162$ and $\operatorname{rns}(G)=(0,0,0,0,162,0,0,0,0,0)$.

(ii) $\left|E S S_{4}(G)\right|=\left|E S S_{6}(G)\right|=15$ and ens $(G)=(0,0,0,15,0,15,0,0,0,0)$, therefore

$\operatorname{Edim}(G)=4$.

(iii) $\operatorname{ESS}(G)=\operatorname{DISC}(G)$, therefore $d n s(P e t)=(0,0,0,15,0,15,0,0,0,0)$.

Proof. ( $i$ ) By Theorem 6.6 all the reducts of the Petersen graph have cardinality 5. In the proof of the same Theorem we counted such subsets. They are in total 162 . So $(i)$ holds.

(ii)-(iii) By Theorem 6.6 it follows that all subsets appearing in the discernibility matrix $\Delta[G]$ are also minimal elements of the poset $\mathbb{D}(G)$. Let us note now that all the 6subsets that appear in the places of $\Delta[G]$ are mutually different between them. In fact, if $B$ is such a subset, by Theorem $6.6, G[B]$ is isomorphic to $H$ and it is easy to see that $B=\Delta_{G}(x, y)$, where $x, y \in B$ are the vertices having degree 3 in $G[B]$. Thus there are exactly 15 of these subsets. By Proposition 6.4, a subset $A \in D I S C_{4}(G)$ is the complement in $V(G)$ of a subset $B \in D I S C_{6}(G)$. Thus $\left|D I S C_{4}(G)\right|=15$ and the thesis follows.

Example 6.12. Let $G=$ Pet. Referring to Figure 1 in the introductory section, we can use the classification result obtained in Theorem 6.6 to easily determine when a vertex subset $W$ of $G$ is a reduct. For example, if we take $A=\{1,5,6,8,10\}$ then $A$ is a reduct of $G$ because the subgraph $G[A]$ generated by $A$ has the form $(a)$ in the statement of Theorem 6.6. Analogously, the vertex subsets $\{1,3,6,7,9\},\{2,3,4,5,6\}$, $\{1,2,3,4,6\}$ are all reducts of $G$ because their generated subgraphs are respectively of the type $(b),(c),(d)$ in the statement of Theorem 6.6. On the other hand, the vertex subset $A=\{6,7,8,9,10\}$ is not a reduct of $G$ because the generated subgraph $G[A]$ is the inner star of $G$, and therefore it is not isomorphic to any graphs among the types $(a),(b),(c),(d)$ in the statement of Theorem 6.6.

\section{Conclusions}

By interpreting the adjacency matrix of a graph as an information table, we were able to connect graph theory with granular computing. The interest of this link is twofold: using granular techniques on graphs and vice versa, ideas that arise in graph theory can be used in granular computing and in the specific case of this paper on data tables managed through rough set theory. By the results and the prospectives opened here, we hope to have shown to the reader that the study of graph theory by means of GrC-RS methods can be an interesting field of study and that both areas can benefit from each other.

For the future, several directions are worth to be explored: apply the ideas of the paper to other classes of graphs; use other techniques from granular computing to analyze graphs and hypergraphs; analyze the meaning of the new introduced concepts in case of a generic table, in particular the extended core in order to exploit it in data mining applications. 


\section{References}

[1] C. Berge, Hypergraphs: Combinatorics of Finite Sets, Elsevier, Amsterdam, 1984.

[2] F.M. Bianchi, L. Livi, A. Rizzi, A. Sadeghian, A Granular Computing approach to the design of optimized graph classification systems, Soft Computing, 18, 2014, 393-412

[3] G. Cattaneo, G. Chiaselotti, D. Ciucci, T. Gentile, On the connection of Hypergraph Theory with Formal Concept Analysis and Rough Set Theory. Information Sciences, Available online 16 October 2015, doi:10.1016/j.ins.2015.09.054.

[4] J. Chen, J. Li, An application of rough sets to graph theory, Information Science, 201, 2012, 114-127.

[5] G. Chen, N. Zhong, Granular Structures in Graphs, Proc. RSKT 2011, Lecture Notes in Computer Science, 6954, 2011, 649-658

[6] G. Chen, N. Zhong, Three Granular Structure Models in Graphs, Proc. RSKT 2012, Lecture Notes in Computer Science, 7414, 2012, 351-358,

[7] G. Chen, N. Zhong, Y. Yao, A Hypergraph Model of Granular Computing, Proc. IEEE Int. Conf. on Granular Computing 2008, 130-135

[8] G. Chiaselotti, D. Ciucci, T. Gentile, Simple Undirected Graphs as Formal Contexts, Proc. ICFCA, Lecture Notes in Computer Science, Volume 9113, 2015, 287-302.

[9] G. Chiaselotti, D. Ciucci, T. Gentile, F. Infusino, Rough Set Theory Applied to Simple Undirected Graphs. Proc. RSKT 2015, Lecture Notes in Computer Science, Vol. 9436, pp. 423-434, Springer 2015

[10] G. Chiaselotti, D. Ciucci, T. Gentile, F. Infusino, Preclusivity and Simple Graphs. Proc. RSFDGrC 2015, Lecture Notes in Computer Science, Vol. 9437, 127-137, Springer 2015

[11] G. Chiaselotti, T. Gentile, F. Infusino, P.A. Oliverio, Rough Sets for $n$-Cycles and $n$-Paths. Applied Mathematics and Information Sciences, (To Appear).

[12] G. Chiaselotti, D. Ciucci, T. Gentile, F. Infusino, Generalizations of Rough Set Tools Inspired by Graph and Hypergraph Theory, submitted to Fundamenta Informaticae, 2015

[13] R. Diestel, Graph Theory (4th edition), Graduate Text in Mathematics, Springer 2010.

[14] P. Doreian, V. Batagelj, A. Ferligoj, Generalized Blockmodeling. Cambridge University Press, 2005

[15] T. Eiter and G. Gottlob, Identifying the Minimal Transversals of a Hy- pergraph and Related Problems,SIAM J. COMPUT., 24, 1995, 1278-1304

[16] T. Eiter and G. Gottlob, Hypergraph transversal computation and related problems in logic and AI, Proc. JELIA 2002, Lecture Notes in Computer Science, Volume 2424, 2002, 549-564

[17] B. Ganter, R. Wille, Formal Concept Analysis. Mathematical Foundations, Springer-Verlag, 1999.

[18] C. Godsil, G. Royle, Algebraic Graph Theory, Graduate Text in Mathematics, Springer 2001.

[19] P. Hońko, Relational pattern updating, Information Science, 189, 2012, 208-218.

[20] P. Hońko, Association discovery from relational data via granular computing, Information Science, 10, 2013, 136-149.

[21] X. Kang, D. Li, S. Wang, K. Qu, Formal concept analysis based on fuzzy granularity base for different granulations, Fuzzy Sets and Systems, 203, 2012, 33-48.

[22] V Kreinovich, Interval Computation as an Important Part of Granular Computing: An Introduction, in [34], 2008, 3-32

[23] Lee T. T., An Information-Theoretic Analysis of Relational Databases - part I: Data Dependencies and Metric. IEEE Transactions on Software Engineering SE-13 (1987), pp. 1049-1061.

[24] M. Liang, B. Liang, L. Wei, X. Xu, Edge rough graph and its application. IEEE Fuzzy Systems and Knowledge Discovery (FSKD), Volume 1, 2011 Eighth International Conference.

[25] T. Y. Lin, Data Mining: Granular Computing Approach, in Methodologies for Knowledge Discovery and Data Mining Lecture Notes in Computer Science, 1574, 1999, 24-33.

[26] T. Y. Lin, Data Mining and Machine Oriented Modeling: A Granular Approach, Applied Intelligence, 13, 2000, 113-124.

[27] Z. Pawlak, Information systems theoretical foundations, Information Sciences 6, Issue 3, 1981, pp. 205-218

[28] Pawlak Z., Rough sets. Theoretical Aspects of Reasoning about Data. Kluwer Academic Publisher, 1991.

[29] Z. Pawlak, A. Skowron, Rudiments of rough sets, Information Sciences 177, 2007, pp. 3-27

[30] Z. Pawlak, A. Skowron, Rough sets: Some extensions, Information Sciences 177, 2007, pp. 28-40

[31] Z. Pawlak, A. Skowron, Rough sets and Boolean reasoning, Information Sciences 177, 2007, pp. 41-73

[32] W. Pedrycz, Granular Computing: An Emerging Paradigm, Springer-Verlag, Berlin (2001). 
[33] W. Pedrycz, K. Hirota, W. Pedrycz, F. Dong, Granular representation and granular computing with fuzzy sets, Fuzzy Sets and Systems, 203, 2012, 17-32.

[34] W. Pedrycz, A. Skowron, V. Kreinovich, Handbook of Granular Computing, Wiley, 2008

[35] T. Qiu, X. Chen, Q. Liu, H. Huang, Granular Computing Approach to Finding Association Rules in Relational Database, International Journal of Intelligent Systems, 25, 2010, 165-179.

[36] A. Skowron, C. Rauszer, The Discernibility Matrices and Functions in Information Systems, Intelligent Decision Support, Theory and Decision Library series, vol. 11, Springer Netherlands, 1992 pp. 331-362.

[37] A. Skowron, P. Wasilewski, Information systems in modeling interactive computations on granules, Theoretical Computer Science, 412, 2011, 5939-5959.

[38] A. Skowron, P. Wasilewski, Interactive information systems: Toward perception based computing, Theoretical Computer Science, 454, 2012, 240-260.

[39] J. G. Stell, Granulation for Graphs, Sp. Inf. Th., Lecture Notes in Computer Science, Volume 1661, 1999, 417-432

[40] J. G. Stell, Relations in Mathematical Morphology with Applications to Graphs and Rough Sets, Sp. Inf. Th., Lecture Notes in Computer Science, Volume 4736, 2007, 438-454.

[41] J. G. Stell, Relational Granularity for Hypergraphs, RSCTC, Lecture Notes in Computer Science, Volume 6086, 2010, 267-276.

[42] J. G. Stell, Formal Concept Analysis over Graphs and Hypergraphs. Proc. GKR 2013, Lecture Notes in Computer Science, Volume 8323, 2014, 165-179

[43] J. Stepaniuk, Rough - Granular Computing in Knowledge Discovery and Data Mining, 2008 Springer-Verlag Berlin-Heidelberg.

[44] J. Wang, W. Zhu, An approach to covering-based rough sets through bipartite graphs, Proc. FUZZIEEE, 2014, 1213-1218

[45] S. Wang, Q. Zhu, W. Zhu, F. Min, Equivalent Characterizations of Some Graph Problems by Covering-Based Rough Sets, Journal of Applied Mathematics, Volume 2013, Article ID 519173, 7 pages.

[46] W. Z. Wu, Y. Leung, J. S. Mi, Granular Computing and Knowledge Reduction in Formal Contexts, IEEE Trans. on Knowledge and Data Engineering, 21, 2009, 1461-1474

[47] Y. Y. Yao, On modeling data mining with granular computing, COMPSAC 2001. IEEE, 2001, pp. $638-643$.

[48] Y. Y. Yao, Information granulation and rough set approximation, International Journal of Intelligent Systems, 2001 Vol. 16, No. 1, 87-104.

[49] Y.Y. Yao, A Partition Model of Granular Computing, in Transactions on Rough sets I Lecture Notes in Computer Science, vol. 3100, Springer-Verlag, 2004, pp. 232-253.

[50] Y. Y. Yao, N. Zhong, Granular Computing using Information Tables, in Data Mining, Rough Sets and Granular Computing, Physica-Verlag, 2002, pp. 102-124.

[51] J. T. Yao, Y. Y. Yao, A Granular Computing Approach to Machine Learning, in Proceedings of the 1st International Conference on Fuzzy Systems and Knowledge Discovery, 2002, 732-736

[52] L.A. Zadeh, Fuzzy sets and information granularity, in: Advances in Fuzzy Set Theory and Applications, Gupta, N., Ragade, R. and Yager, R. (Eds.), North- Holland, Amsterdam, 3-18, 1979.

[53] L. A. Zadeh, Towards a theory of fuzzy information granulation and its centrality in human reasoning and fuzzy logic, Fuzzy Sets and Systems, 19, 1997, 111-127. 